\title{
A lateralized alerting deficit in left-brain-damaged patients
}

\author{
MARTIN ARGUIN \\ Montreal Neurological Institute, Montreal, Quebec, Canada \\ PATRICK CAVANAGH \\ Harvard University, Cambridge, Massachusetts \\ and \\ YVES JOANETTE \\ Centre Hospitalier Côte-des-Neiges \\ and Faculté de Médecine, University of Montreal, Montreal, Quebec, Canada
}

\begin{abstract}
Any warning stimulus preceding a visual target generally leads to progressive reduction in response times with increases in the warning-to-target temporal interval. This effect, called alerting, seems to be correlated with cortical activation. Three visuospatial cuing experiments were conducted with 10 non-neglecting left-brain-damaged patients in order to examine the possibility of an alerting impairment following lesions of the left hemisphere. Results indicate that large left-hemisphere lesions lead to a delay in the alerting effect of a warning signal for the processing of contralesional targets. Moreover, the results suggest that a lowered alerting state of the damaged hemisphere leads to a refractory difficulty in disengaging the focus of spatial attention from an invalidly cued ipsilesional location.
\end{abstract}

Visual attention disorders are common after brain damage. Posner and collaborators, as well as other researchers, have made extensive use of the visuospatial cuing paradigm in documenting the nature of the selective attention deficits present in various brain-damaged populations ( $\mathrm{Ar}-$ guin \& Bub, 1993; Baynes, Holtzman, \& Volpe, 1986; Morrow \& Ratcliff, 1988; Posner, Cohen, \& Rafal, 1982; Posner, Inhoff, Friedrich, \& Cohen, 1987; Posner, Rafal, Choate, \& Vaughan, 1985; Posner, Walker, Friedrich, \& Rafal, 1984, 1987; Rafal \& Posner, 1987; Rafal, Posner, Friedman, Inhoff, \& Bernstein, 1988). In the visuospatial cuing task, subjects are shown a cue that indicates the most probable location of an incoming visual target to which they are to respond as rapidly as possible. In such experiments, even in the absence of shifts of ocular fixation, normal individuals typically show shorter re-

The research reported here was conducted for partial fulfillment of the first author's PhD degree and was led at the Département de Psychologie, Université de Montréal and at the Laboratoire TheophileAlajouanine, Centre Hospitalier Côte-des-Neiges. We thank Michael Posner and other, anonymous reviewers, for their comments on a previous version of this manuscript. This work was supported by a graduate scholarship from the Conseil de Recherches Médicales du Canada (CRMC) to M.A., by Grant A8606 from the National Science and Engineering Research Council of Canada to P.C., and by Grant PG-28 from the CRMC to Y.J. Y.J. is Scientist of the CRMC. The order of the second and third authors was determined by the toss of a coin. Correspondence should be addressed to M. Arguin, Neurolinguistics, Montreal Neurological Institute, 3801 University, Montreal, PQ, Canada H3A 2B4 (e-mail: mi51@musica.mcgill.ca). sponse times (RTs) if the cue indicates the true target location (valid cue) than if the target is presented at another location (invalid cue-see, among others, Egly \& Homa, 1984; Eriksen \& Hoffman, 1973, 1974; Hughes \& Zimba, 1985; Jonides, 1980, 1981; Klein, 1980; Müller \& Rabbitt, 1989; Posner, 1980; Posner, Nissen, \& Ogden, 1978; Rizzolatti, Riggio, Dascola, \& Umiltà, 1987; Shulman, Remington, \& McLean, 1979; Tassinari, Agliotti, Chelazzi, Marzi, \& Berlucchi, 1987). This result is taken as evidence that subjects can covertly shift the spatial allocation of a focus of selective attention.

The use of the spatial cuing paradigm to study braindamaged subjects has revealed a variety of deficits that affect the basic operations involved in selective spatial attention. An impairment in moving the attention focus from one location to another has been reported in patients with progressive supranuclear palsy, who particularly suffer from damage to the superior colliculus (Posner et al., 1982; Rafal et al., 1988). In contrast, patients with thalamic damage have exhibited deficits in engaging their attention to targets located on the contralesional side of their lesions (Rafal \& Posner, 1987; see also Petersen, Robinson, \& Morris, 1987, for relevant observations in the monkey). Finally, subjects with parietal damage have been found to suffer from a difficulty in disengaging their attention from a particular location when it next needs to be shifted toward the contralesional hemifield (Arguin \& Bub, 1993; Baynes et al., 1986; Morrow \& Ratcliff, 1988; Posner et al., 1982; Posner, Inhoff, et al., 1987; Posner et al., 1984; Posner, Walker, et al., 1987). These 
impairments in basic spatial attention operations (i.e., moving, engaging, and disengaging) are dissociable from one another, and each deficit is characterized by a particular pattern of performance in the visuospatial cuing task. These patterns will be discussed in detail later.

Although the cuing task is mainly designed for studying the spatial orienting of visual selective attention, it can be used for other purposes. Intrinsic to the paradigm is the warning signal provided by the onset of the cue as to the imminence of the target. Such a warning signal is known to produce in subjects a spatially nonspecific preparation to respond to a perceptual event, an effect called alerting (Posner, 1978; Posner \& Boies, 1971) or activation (Heilman \& van den Abell, 1979, 1980; Pribram \& McGuinness, 1975). The effect of a warning signal on the alertness level of the subject can be observed from the brain's electrical activity. Following the onset of a warning signal, EEG recordings show a temporary blocking of alpha activity, which is replaced by fast desynchronized activity (Lansing, Schwartz, \& Lindsley, 1959) and a slow increase in negativity called the contingent negative variation (Donchin, Coles, \& Gratton, 1984; Walter, Cooper, Aldridge, McCallum, \& Winter, 1964). Behaviorally, the effect of alerting is made apparent by decreasing RTs to the target with an increase in the temporal interval following the warning signal until an asymptotic level of performance is reached about $200-500 \mathrm{msec}$ after the onset of the warning signal (Bertelson, 1967; Lansing et al., 1959; Posner, 1978; Posner \& Boies, 1971; Posner, Inhoff, et al., 1987; Posner, Klein, Summers, \& Buggie, 1973; Sanders, 1972; Thomas, 1974). The time course of the alerting effect on RTs is roughly correlated with the EEG changes that occur after the onset of a warning signal (Gaillard \& Näätänen, 1973; Hillyard, 1969; Lansing et al., 1959). When the task involves a response choice, the reduction of RTs following a warning signal may be accompanied by an increase in error rate (i.e., speed-accuracy tradeoff; Posner et al., 1973). This has led to the conclusion that alerting does not affect the rate of build-up of perceptual information but rather the rate at which a higher level attention system responds to that build-up or the criterion that the subject sets for response production. This dissociation between the system responsible for alertness and that involved in the buildup of sensory information has been confirmed in several reports (Posner \& Boies, 1971; Posner et al., 1973; Thomas, 1974).

Observations reported by Posner, Inhoff, et al. (1987) have shown that alertness may be impaired by damage to the right cerebral hemisphere. In their experiment, subjects had to produce a simple RT to a visual target presented to the left or right hemifield. This target was preceded by either a valid or an invalid spatial cue, or by no cue at all (the no-cue condition was run in separate blocks). Patients with right-brain damage showed somewhat longer RTs to contralesional than to ipsilesional targets on valid-cue trials (hemifield asymmetry of about 100 msec; Posner, Inhoff, et al., 1987, Figure 1). How- ever, the RT difference between hemifields was dramatically increased in the no-cue condition (RTs: contralesional field $=820 \mathrm{msec}$; ipsilesional field $=525 \mathrm{msec}$; Posner, Inhoff, et al., 1987, Table 2). This large increase in RTs to contralesional targets when no warning signal was presented before the target has been taken as an indication that right-hemisphere damage leads to an impairment in maintaining a high level of alertness during the time interval separating one trial from the next. The presentation of a warning signal before the target served to reinstate a proper alerting level in these patients and thus produced roughly comparable RTs to contra- and ipsilesional targets, at least when the spatial cue was valid. In contrast, patients with left-hemisphere damage only showed a moderate and nonsignificant visual field asymmetry on no-cue trials (RTs: contralesional field = $470 \mathrm{msec}$; ipsilesional field $=430 \mathrm{msec}$; Posner, Inhoff, et al., 1987, Table 2) as well as on valid-cue trials, thus suggesting no particular deficit in maintaining an alert state during the time period separating trials.

This asymmetry in the effect of unilateral brain damage on alertness is congruent with previous results from patients with lesions of the right cerebral hemisphere (De Renzi \& Faglioni, 1965; Heilman, 1985; Làdavas, Del Pesce, \& Provinciali, 1989). It is also congruent with results from normal individuals that indicate a righthemisphere dominance for cortical activation (Heilman $\&$ van den Abell, 1979, 1980). As mentioned above, cortical activation seems to be correlated with the behavioral effects of alerting. Finally, a review of the literature on neurotransmitter action and lateralization by Tucker and Williamson (1984) also supports the hypothesis of a righthemisphere superiority for sustaining alertness.

It appears unlikely, however, that this dominance of the right hemisphere for alertness is absolute (see Heilman \& van den Abell, 1979, 1980, and Tucker \& Williamson, 1984, for relevant evidence). In fact, the data reported by Posner, Inhoff, et al. (1987) in their right-braindamaged patients does suggest that the left hemisphere may contribute to the level of alertness. Thus, the deficit found in these subjects in the no-cue condition was only apparent with contralesional targets. This capacity of rightbrain-damaged patients to show RTs to ipsilesional targets that were within a normal range in the no-cue condition suggests that the intact left hemisphere of these subjects maintained a sufficiently high level of alertness. In turn, this implies that alertness may not be a global condition in these brain-damaged cases, and that each cerebral hemisphere has its own state of alertness; these states are thus more or less separable from each other. Note that this hypothesis agrees with the proposal of several authors that the cerebral hemispheres may serve as two independent pools of attentional resources (Eglin, 1987; Friedman \& Polson, 1981; Hellige, Cox, \& Litvac, 1979; Kinsbourne, 1970, 1975, 1977, 1978, 1987; Kinsbourne \& Hicks, 1978; Reuter-Lorenz, Kinsbourne, \& Moscovitch, 1990).

In support of the conjecture presented above, we report a series of three visuospatial cuing experiments in 
which we found an impairment in the time course of (i.e., delayed) alerting for contralesional targets in a subset of the 10 left-brain-damaged subjects tested. ${ }^{1}$ This alerting deficit was characterized by elevated RTs to contralesional targets at short warning-to-target temporal intervals, with a marked reduction of this hemifield asymmetry at longer temporal intervals. The evidence reported below also suggests, in support of Posner's view about the interactions between attentional systems (Posner, 1990; Posner, Inhoff, et al., 1987), that the alerting disorder may affect the operations involved in the spatial allocation of selective attention. Finally, anatomical investigations will provide indications regarding the feature of lesions associated with the alerting impairment.

The same basic design was used in all three experiments. The targets to which the subjects had to produce a simple RT were presented to the left or right visual hemifield and were preceded by valid or invalid spatial cues (which also acted as warning signals). In order to study the time course of the effects of these cues/warning signals, the temporal interval separating them from the subsequent targets was varied (stimulus onset asynchronies-SOAs-of 50, 150,600, or $1,000 \mathrm{msec}$ ).

Each experiment differed from the others in the type of spatial cue/warning signal that was used. The spatial cues were varied in this way because previous results regarding the spatial orienting of attention had indicated that the physical nature of the cuing stimulus might not be neutral with respect to hemifield asymmetries in RTs in braindamaged patients. For instance, results reported by Posner et al. (1984) indicated that parietal patients with a disorder affecting the disengagement of selective attention showed longer RTs to contralesional targets at short SOAs following valid central cues (arrowhead shown at fixation) but not following valid luminance cues (unilateral brightening of a box surrounding the target location; also called peripheral cues). Since the main aspect of the results that we intended to consider in each experiment was the variation of hemifield asymmetries as a function of the temporal interval between the cue/warning and the target, it appeared that the use of a single type of cue/warning might have led to results that did not allow one to separate effects related to attentional alerting from those related to the spatial orienting of selective attention. The spatial cues/warning signals used in the present experiments were thus presented at fixation (central cue; Experiment 1), bilaterally (the cue was the direction of motion-toward the left or right-of peripheral gratings; Experiment 2), or unilaterally (luminance cue; Experiment 3).

\section{METHOD}

\section{Subjects}

Inclusion as well as exclusion criteria were applied for subject selection in order to control factors that otherwise might have rendered the interpretation of the results equivocal, and to make sure that subjects were able to perform the tasks adequately. The inclu- sion criteria were (1) right handedness, as assessed with the Edinburgh Handedness Inventory (score between +80 and +100 ; Oldfield, 1971); and (2) brain lesion of vascular origin, a first occurrence of brain damage, with a unique lesion lateralized to one hemisphere (applies only to brain-damaged subjects). The exclusion criteria were (1) reduced and uncorrected visual acuity; (2) ocular disease such as glaucoma or cataracts; (3) visual field deficits, as assessed by campimetry (this applies only to brain-damaged subjects); (4) visual hemineglect, as assessed with the bells test (Gauthier, Dehaut, \& Joanette, 1989; this applies only to braindamaged subjects); and (5) an aphasia that may impair the understanding of instructions (this applies only to brain-damaged subjects).

Following the application of the selection criteria, a group of 10 left-brain-damaged subjects whose mean age was 65 years was examined. The minimum time period between lesion onset and testing was 2 months. A second group of 10 neurologically intact subjects whose mean age was 71 years served as controls.

\section{Materials}

The experiments were controlled by an Amiga microcomputer, and the stimuli were displayed on an RGB monitor located $57 \mathrm{~cm}$ from the subjects. A chin rest prevented head movement. The subjects used a computer mouse to respond. The experiments were run in a dimly lit room.

An ocular fixation control was used to ensure that the stimuli were displayed in the proper visual hemifield. This was achieved with the use of a pupil and corneal reflection tracking system (ISCAN, Model RK-426) linked to the control computer. This tracking system analyzes video images obtained from a black and white camera equipped with an infrared filter. An infrared source served for illumination. Trials began only when the subject fixated a central point. If, during a trial, the subject shifted his/her ocular fixation toward the target location (displayed left or right of fixation) so that the distance between ocular fixation and the stimulus was under $3.5^{\circ}$ of visual angle, that trial was immediately terminated and run again later in the session. Across experiments and groups, between $1.0 \%$ and $2.7 \%$ of the trials were eliminated on this criterion.

\section{Stimuli}

A white fixation stimulus (luminance of about $61 \mathrm{~cd} / \mathrm{m}^{2}$ ) was shown at the center of the display screen between trials. It was made of a $0.2^{\circ}$ dot surrounded by a $1.3^{\circ}$ empty circle. The subjects were instructed to keep their eyes directed toward the fixation location as much as possible throughout the experiment.

Two empty squares $\left(3.1^{\circ}\right.$ wide $\times 2.8^{\circ}$ high) were displayed permanently on the screen. They were presented left and right of the fixation location, and the center-to-center distance between them and the fixation stimulus was $10.6^{\circ}$ of visual angle. In Experiments 1 and 2, these squares remained white (luminance, about $61 \mathrm{~cd} / \mathrm{m}^{2}$ ) throughout the session. In Experiment 3 , these squares were gray (luminance, about $6 \mathrm{~cd} / \mathrm{m}^{2}$ ) between trials. The target was a white filled circle $\left(0.8^{\circ}\right.$ in diameter), centered within one of the two squares, and was displayed for a duration of $200 \mathrm{msec}$.

In Experiment 1 (central cue), the spatial cue/warning signal was an arrow $\left(1.6^{\circ}\right.$ wide $\times 1.0^{\circ}$ high $)$, shown at fixation, which pointed either left or right. In Experiment 2 (motion cue), the warning signal was the onset of square wave gratings $\left(2.9^{\circ}\right.$ wide $\times 1.9^{\circ}$ high, spatial frequency of $0.7 \mathrm{cycles} / \mathrm{deg}$ ) displayed bilaterally at a distance of $1.4^{\circ}$ above and below the squares in which the target could appear. The spatial cuing property of this signal consisted in the direction (left or right) toward which the lines in the gratings moved (speed of movement of $5.0 \mathrm{cycles} / \mathrm{sec}$ ). In Experiment 3 (luminance cue), the cue/warning was the illumination of one of the peripheral squares. The luminance of the lighted square thus changed from $6 \mathrm{~cd} / \mathrm{m}^{2}$ to $61 \mathrm{~cd} / \mathrm{m}^{2}$. 
In all experiments, the spatial cue/warning signal remained visible until the end of the trial. The subjects were instructed to use the information provided by the cue by directing their attention toward the cued location while fixating the center of the screen ("to look out the corner of their eyes").

\section{Procedure}

The subjects' task was to respond as rapidly as possible to the onset of the target. This response was made by pressing the mouse button with the index finger. All subjects were required to use the left hand to respond, since many (5 out of 10 ) left-brain-damaged subjects suffered from a right-arm plegia or paresis. All responses made between 150 and 3,000 msec after target onset were immediately followed by auditory feedback that consisted of a high-pitched sound. Responses made less than $150 \mathrm{msec}$ or more than 3,000 msec after target onset were followed by a low-pitched sound. These trials were eliminated and run again later in the session. Across groups and experiments, between $0.0 \%$ and $2.6 \%$ of trials were eliminated on this criterion.

On each trial, a spatial cue/warning signal preceded target onset by a stimulus onset asynchrony (SOA) of $50,150,600$, or $1,000 \mathrm{msec}$. The spatial cue was valid on $80 \%$ of the trials and invalid on $20 \%$ of the trials. The subjects were informed of these probabilities when given the instructions.

Each experiment comprised 16 conditions resulting from variations along three factors. These were: cue validity (valid or invalid), SOA between cue and target $(50,150,600$, or $1,000 \mathrm{msec})$, and hemifield in which the target was displayed (left or right). Conditions were distributed according to a pseudorandom scheme, with 240 experimental trials per session per subject. The experimental session was immediately preceded by 35 training trials. RT was the dependent variable.

\section{RESULTS}

The main purpose of the present report is to investigate whether an alerting impairment may be found in subjects with unilateral left brain lesions. However, an a priori assumption of homogeneity of our brain-damaged sample as to the presence or absence of a deficit of attentional alerting is hardly tenable. The brain-damaged subjects were not selected for the location or size of their lesions, and these parameters could be expected to affect the occurrence of the deficit. Moreover, there is at present no criterion based on the properties of lesions that allows one to predict the presence of the impairment in any specific case. For these reasons, the first phase of data analysis was an attempt to identify individual brain-damaged subjects who exhibited a pattern of performance congruent with an alerting impairment.

What is the RT pattern that is to be expected from a patient with an alerting deficit for contralesional stimuli? Our basic assumption in the present work is that such an impairment should reveal itself by a delay in the alerting effect of a warning signal for a contralesional target relative to an ipsilesional one. In this case, longer RTs to contralesional than to ipsilesional targets should be observed at short warning-to-target temporal intervals (in the present experiments, SOAs of 50 and $150 \mathrm{msec}$ ). This RT asymmetry between hemifields should, however, be absent or become smaller at longer SOAs following the warning signal (SOAs of 600 and $1,000 \mathrm{msec}$ ), at which point the alerting effect should have reached asymptote in a normal hemisphere (Bertelson, 1967; Lansing et al., 1959; Posner, 1978; Posner \& Boies, 1971; Posner, Inhoff, et al., 1987; Posner et al., 1973; Sanders, 1972; Thomas, 1974). This interaction between SOA and hemifield was used as a preliminary indicator for a disorder affecting the time course of alerting in individual cases.

Only the observations from valid-cue trials were used for the phase of individual data analyses: Previous observations on spatial cuing in brain-damaged subjects had indicated that cue validity may interact with hemifield asymmetries in RTs in a wide variety of brain-damaged subjects (Baynes et al., 1986; Morrow \& Ratcliff, 1988; Posner et al., 1982; Posner, Inhoff, et al., 1987; Posner et al., 1984; Posner, Walker, et al., 1987; Rafal \& Posner, 1987), and the number of observations for each SOA $X$ hemifield combination in individual subjects was quite small on invalid-cue trials $(n=6)$.

A measure of asymmetry reflecting an SOA $\times$ hemifield interaction was thus computed from the individual median RTs in valid-cue conditions. The following formula was used to calculate this measure:

$$
\begin{aligned}
M & =\left[\left(R_{50}-L_{50}\right)+\left(R_{150}-L_{150}\right)\right] \\
& -\left[\left(R_{600}-L_{600}\right)+\left(R_{1,000}-L_{1,000}\right)\right],
\end{aligned}
$$

where the letters $R$ and $L$ represent RTs with right- and left-hemifield targets, respectively, and the numerical indexes represent the SOA between cue and target.

A high absolute value of the measure of asymmetry signifies an interaction of SOA $\times$ hemifield. As indicated above, this interaction is the pattern of results that is expected from subjects with impaired alerting for contralesional stimulation. Specifically, these subjects should show a large hemifield asymmetry in RTs-longer for a contralesional target-on valid-cue trials at short cue-totarget SOAs, and a reduction of this asymmetry at later SOAs. Also, a positively signed measure of asymmetry is consistent with impaired alerting for right-hemifield stimuli while a negatively signed measure of asymmetry is consistent with impaired alerting for left-hemifield stimuli. Any left-brain-damaged subject exhibiting a positively signed measure of asymmetry of which the absolute value was larger than three standard deviations above the mean of the absolute values of the measures of asymmetry obtained by the control subjects on the same task was considered to show preliminary evidence of an alerting deficit.

\section{Individual Data Analyses}

Experiment 1: Central cue. Figure 1 presents the outcome of the analysis of the individual asymmetry data with valid central cues. Six of the normal controls showed positively signed measures of asymmetry, and 4 received negative values. Normal controls (Figure 1A) showed an average asymmetry value $(3.4 \mathrm{msec})$ that did not differ significantly from zero $[t(9)=0.2, \mathrm{n} . \mathrm{s}$.]. In contrast, 5 of the 10 brain-damaged subjects (P6-P10) exhibited very large measures of asymmetry (Figure 1B). ${ }^{2}$ The absolute value of each of these measures in subjects P6-P10 was larger than three standard deviations above the mean for 

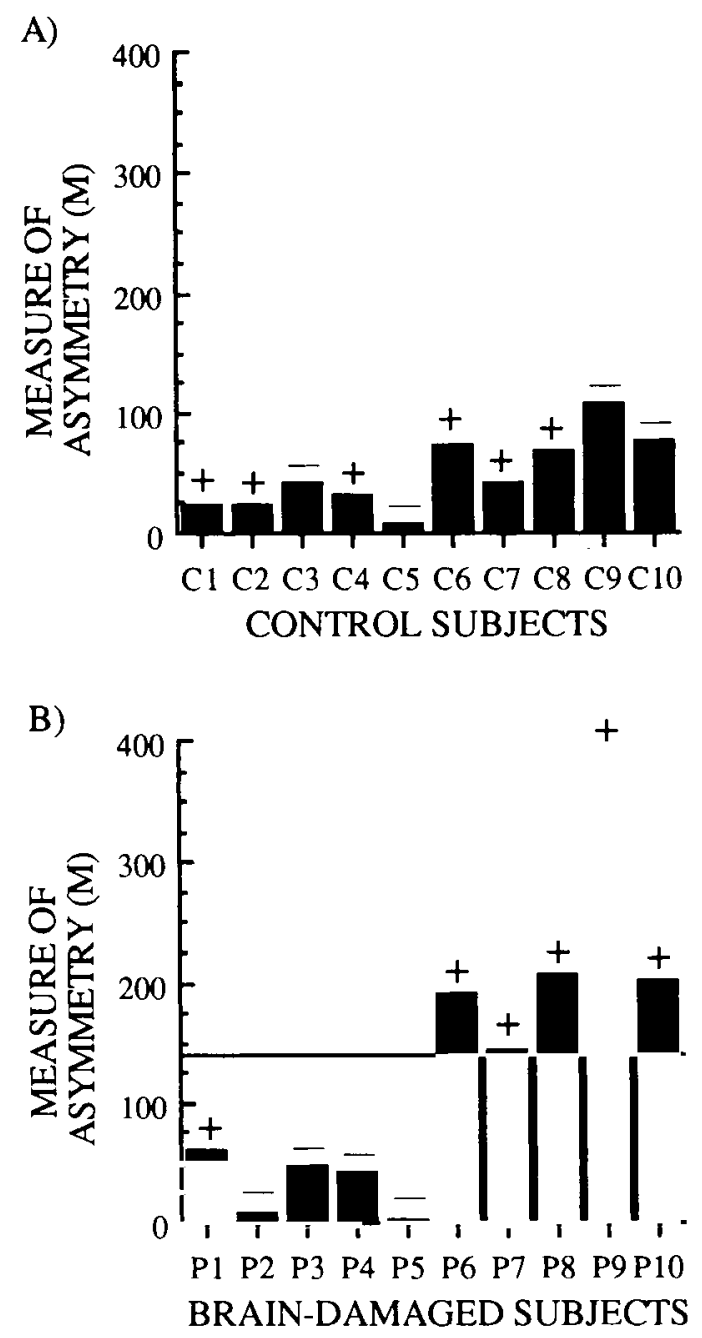

Figure 1. Measures of asymmetry $(M)$ observed in each subject in Experiment 1 (central cue). The "+" or " -" above each column indicates the sign of $M$ (positive or negative). (A) Control subjects (C1-C10). (B) Brain-damaged subjects (P1-P10). The horizontal line in this histogram represents the value of three standard deviations above the mean observed in control subjects.

the control subjects. Moreover, the sign of the measure for each of these brain-damaged subjects was positive, which is consistent with a left-hemisphere lesion (see above). These results for Subjects P6-P10 reflect large RT asymmetries (average of $279.7 \mathrm{msec}$, left part of Equation 1) after the presentation of a valid central cue at short SOAs-with RTs longer with a contralesional target-but smaller asymmetries (average of $53.0 \mathrm{msec}$, right part of Equation 1) at longer SOAs.

Following the rationale upon which this analysis was performed, these results suggest that 5 of the braindamaged subjects in our sample (Subjects P6-P10) showed delayed alerting for contralesional stimuli following the presentation of a spatially valid cue/warning signal at fixation. In contrast to this subgroup of brain-damaged cases, Subjects P1-P5 obtained measures of asymmetry that were within the range of those observed in the normal controls. Consequently, it appears that Subjects P1-P5 did not suffer from any alerting deficit.

Experiment 2: Motion cue. Individual asymmetry data for valid motion cue trials are presented in Figure 2. Half the control subjects (Figure $2 \mathrm{~A}$ ) showed a positive asymmetry measure and half a negative value. The mean of the asymmetry data for these subjects was $-20.2 \mathrm{msec}$, which did not differ significantly from zero $[t(9)=-1.0$, n.s.]. However, Brain-Damaged Subjects P6-P10 exhibited an abnormally high measure of asymmetry in comparison with the normal controls (Figure 2B). The average RT asymmetries-with longer RTs for right-hemifield targets-shown by these subjects were $346.5 \mathrm{msec}$ at the shorter SOAs and $165.1 \mathrm{msec}$ at longer SOAs. The sign of the measure was positive, which is consistent with a
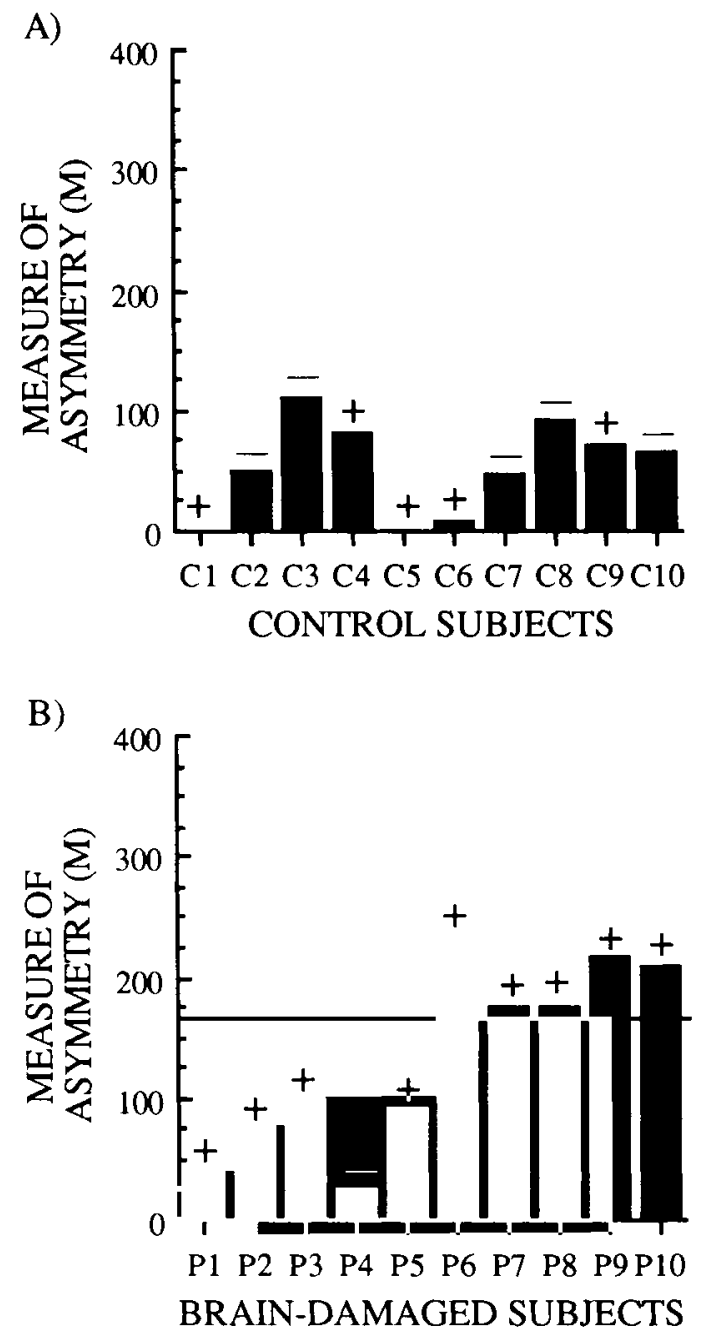

Figure 2. Measures of asymmetry $(M)$ observed in each subject in Experiment 2 (motion cue). (A) Control subjects (C1-C10). (B) Brain-damaged subjects (P1-P10). Conventions are the same as in Figure 1. 

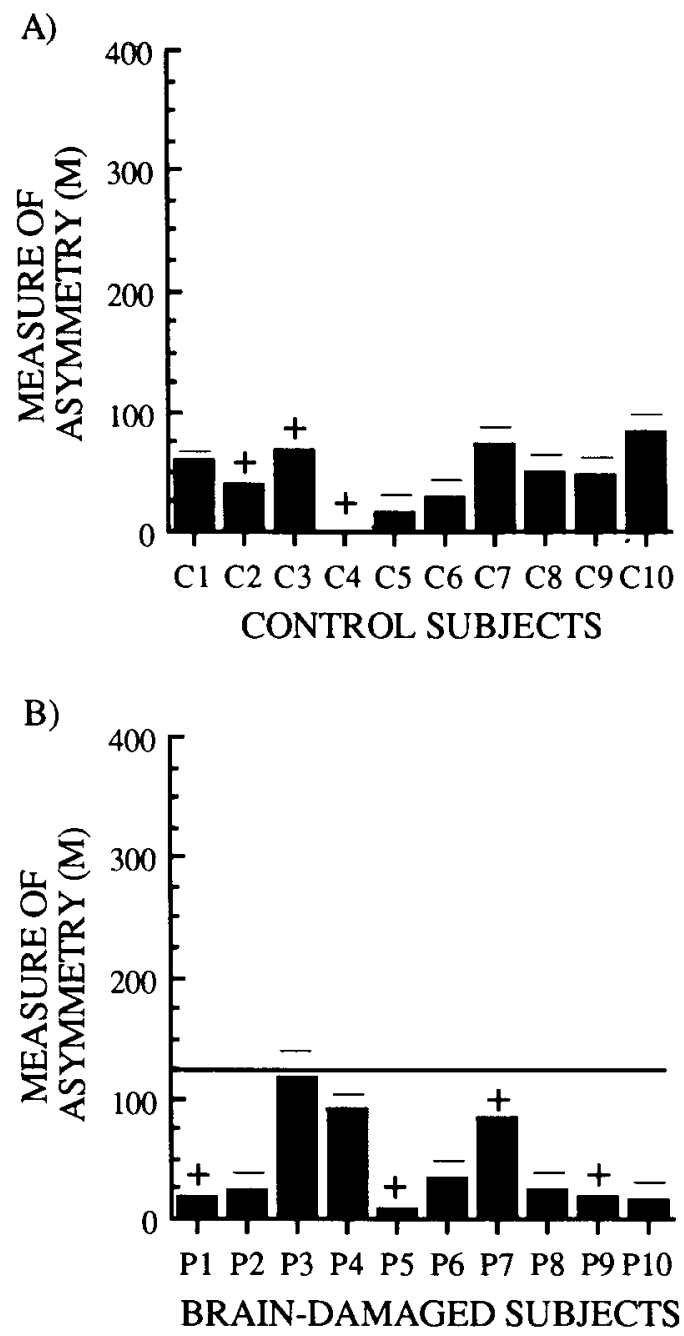

Figure 3. Measures of asymmetry $(M)$ observed in each subject in Experiment 3 (luminance cue). (A) Control subjects (C1-C10). (B) Brain-damaged subjects (P1-P10). Conventions are the same as in Figure 1.

left hemispheric lesion. An important point to note here is that these subjects are the same as those who exhibited a deficit in Experiment 1. Moreover, Subjects P1-P5 showed, as they did in Experiment 1, results comparable to those from the control subjects. Thus, the individual data analyses performed on the results of Experiments 1 and 2 converge in suggesting an impaired time course of alerting for right-hemifield stimulation in Brain-Damaged Subjects P6-P10.

Experiment 3: Luminance cue. Individual asymmetry data for valid luminance cue trials are presented in Figure 3. Control subjects (Figure 3A) showed small asymmetries, which were either positive ( 3 subjects) or negative (7 subjects). As was the case in the previous experiments, the mean of the asymmetry data for control subjects $(-24.7 \mathrm{msec})$ did not differ significantly from zero $[t(9)=-1.6, \mathrm{n} . \mathrm{s}$.$] . None of the brain-damaged subjects$ obtained a measure of asymmetry higher than the cut-off score of three standard deviations above the mean for the normal controls (Figure 3B). Thus, contrary to the results of the two previous experiments, in which warning signals were presented either at fixation or bilaterally, none of the left-brain-damaged subjects appears to have suffered from impaired alerting for right-hemifield stimulation when the warning signal was presented unilaterally in the target hemifield (i.e., the valid luminance cue).

\section{Group Data Analyses}

The first phase of data analysis served to classify braindamaged subjects into two groups: those who showed a performance consistent with a deficit in the time course of alerting for contralesional stimuli (Subjects P6-P10; henceforth the AD group, for "alerting deficit"), and those who did not exhibit any evidence for an alerting impairment (Subjects P1-P5; the nAD group, for "no alerting deficit'). However, the criterion used to identify individual AD patients may also have been congruent with other types of attention impairments-that is, those affecting the spatial allocation of selective attention-and this mainly because it was based only upon the results from valid-cue trials. Therefore, a second, more detailed analysis was performed on the averaged results of each group (i.e., controls, nAD, and AD), to determine whether the impairment observed in the AD group was truly an impairment of alerting.

The second phase of data analysis thus assessed whether the brain-damaged groups differed from the normal controls in ways that corresponded to performance patterns that might be considered as diagnostics of specific types of attention impairments. This procedure thus served to provide a detailed representation of the attention capacities of each brain-damaged group in comparison with the normal controls. As mentioned in the introduction, each known category of deficit affecting the spatial allocation of selective attention is associated with a particular pattern of performance in the visuospatial cuing task. In the present experiments, these performance patterns can be defined across cue validity (valid vs. invalid), target hemifield (ipsilesional vs. contralesional), and SOA (short vs. long) conditions. Table 1 lists the set of interactions that will be analyzed (they are decomposed into their simple effects if significant) to examine whether any of these patterns of performance occurred in our brain-damaged subjects. ${ }^{3}$ Although the number of tests conducted is fairly high, it must be pointed out that each of the contrasts listed in Table 1 is orthogonal to every other. That is, each test examines an aspect of the data that is independent of the others. The complete set of observations gathered from the AD group across the three experiments will be considered to determine the most appropriate account for their impairment. The same will be done for the results of the nAD group to assess whether they manifest any evidence of an attention impairment, and if so, to establish its nature. 
Table 1

Set of Interaction Patterns in the Visuospatial Cuing Task That Correspond to Specific Attention Impairments and That Were Tested in the Group Data Analyses

\begin{tabular}{|c|c|c|c|c|}
\hline \multirow[b]{2}{*}{ Interaction } & \multicolumn{4}{|c|}{ Attention Impairment } \\
\hline & Move & Engagement & Disengagement & Alerting \\
\hline $\mathbf{G} \times \mathbf{H}$ & & $x$ & & \\
\hline$G \times V$ & $x^{2}$ & & & \\
\hline $\mathrm{G} \times \mathrm{H} \times \mathrm{V}$ & $x^{b}$ & & $x^{a}$ & \\
\hline $\mathbf{G} \times \mathbf{H} \times \mathbf{S}$ & & & & $x^{2}$ \\
\hline $\mathbf{G} \times \mathbf{V} \times \mathbf{S}$ & $x^{c}$ & & & \\
\hline $\mathbf{G} \times \mathbf{H} \times \mathbf{V} \times \mathbf{S}$ & $x^{d}$ & & $x^{b}$ & $x^{b}$ \\
\hline
\end{tabular}

Note-G, group; H, target hemifield; $V$, cue validity; $S$, stimulus onset asynchrony (SOA).

An impairment in moving the focus of attention is associated with an absent (patterns a or b, Table 1) or a delayed (patterns $\mathrm{c}$ or d, Table 1) cue validity effect (Posner et al., 1982; Rafal et al., 1988). That is, an RT advantage for validly cued targets is either entirely absent or else present at longer SOAs than it is in normal subjects. This kind of deficit was previously shown to affect movements of attention along a particular axis, with no effect of direction. If present in our experiments, an impairment of the "move" operation may therefore be expected to appear with targets in either hemifield (patterns a or c, Table 1), although the possibility of a deficit restricted to the contralesional hemifield (patterns b or $\mathrm{d}$, Table 1) will also be considered.

A disorder affecting the engagement of attention on the target is characterized by substantially elevated RTs to contralesional targets. This effect remains relatively constant across all SOAs and is present with both valid and invalid spatial cues (Rafal \& Posner, 1987).

Finally, a deficit in disengaging the focus of attention is signaled by disproportionately long RTs to contralesional targets under invalid-cue conditions (Arguin \& Bub, 1993; Baynes et al., 1986; Morrow \& Ratcliff, 1988; Posner et al., 1982; Posner, Inhoff, et al., 1987; Posner et al., 1984; Posner, Walker, et al., 1987). This effect may either occur across all SOA values (pattern a, Table 1; Posner et al., 1984) or only at short SOAs (pattern b, Table 1; Morrow \& Ratcliff, 1988), depending on the duration with which the disengagement difficulty lasts (sustained vs. refractory) after attention is engaged on a particular location. Both possibilities will be examined.

In the same way as the various impairments affecting the spatial allocation of selective attention are associated with specific performance patterns, a disorder in the time course of alerting for contralesional stimulation is also expected to have its particular impact on performance. As indicated above, an alerting impairment is assumed to result in elevated RTs to contralesional targets at short SOAs and a reduction of this hemifield asymmetry at longer SOAs (pattern a, Table 1). Because alerting is defined as a process that is independent of the spatial allocation of attention, this result should not be affected by the particular location to which subjects attend at the time of target onset. In other words, and in principle, the performance pattern diagnostic of delayed alerting for contralesional stimulation in brain-damaged subjects should be independent of the spatial validity of the cues/warning signals. However, one must also allow for the possibility that the spatial features of the cues/warning signals used affect their alerting properties (see Davidson, Schaffer, \& Saron, 1985, and Reuter-Lorenz et al., 1990, for relevant examples). A difference between the spatial properties of valid and invalid spatial cues only occurs in the experiment carried out with the use of luminance cues (Experiment 3). Thus, the spatial features of central (Experiment 1) and motion (Experiment 2) cues remain constant with changes in cue validity, these being displayed either at fixation or bilaterally. In contrast, luminance cues are unilateral and appear either in the same hemifield as the target (valid) or in the opposite hemifield (invalid). Therefore, in Experiment 3, the occurrence of a group $\times$ validity $\times$ hemifield $\times$ SOA interaction (pattern $b$, Table 1) may not be incompatible with a deficit in the time course of alerting.

Since the distinction between the AD and nAD groups was derived a posteriori from the valid trial data-in particular from the occurrence of an SOA $\times$ hemifield interaction-analyses that involve this selection criterion must be treated with caution. We will identify the results falling in this category.

In addition to the set of interactions described in Table 1, the main effects of cue validity and of SOA were assessed. The validity effect will serve to determine that subjects reacted to the spatial cues presented by shifting their focus of selective attention to the cued location. The SOA analysis will serve to confirm that the alerting effect of the spatial cues did actually occur as expected and thus led to a reduction of RTs.

In this second phase of analyses, we used the MANOVA technique described by O'Brien and Kaiser (1985). The contrasts included to examine the effect of the group factor were: controls versus $\mathrm{nAD}$, and controls versus $\mathrm{AD}$. For the main effect of SOA, the contrasts compared RTs with a 50-msec SOA with each of the other SOA values $(150,600$, and $1,000 \mathrm{msec})$. The interaction effects involving the SOA factor only contrasted short (50- vs. 150 $\mathrm{msec})$ versus long (600- vs. $1,000-\mathrm{msec})$ SOAs. This was done because the different patterns of performance tested did not require more detailed comparisons on the SOA factor and because using only one degree of freedom to test this factor instead of three provided greater power in the statistical tests.

Experiment 1: Central cue. RTs observed in the control, nAD, and AD groups with a central cue are shown in Figure 4. The summary of the analyses of variance is presented in Table 2.

A significant effect of cue validity was observed, with shorter RTs when targets were preceded by valid than by invalid spatial cues. Also, an effect of the time interval between cues and targets was seen, with decreasing RTs as a function of SOA. 


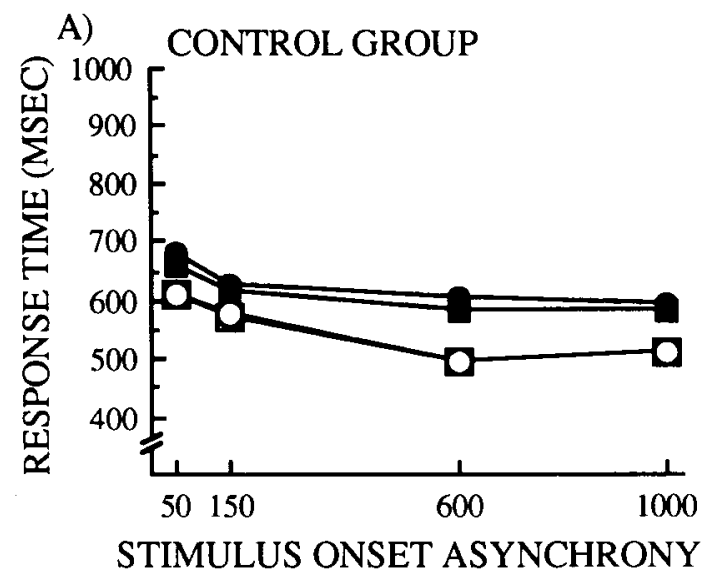

B)

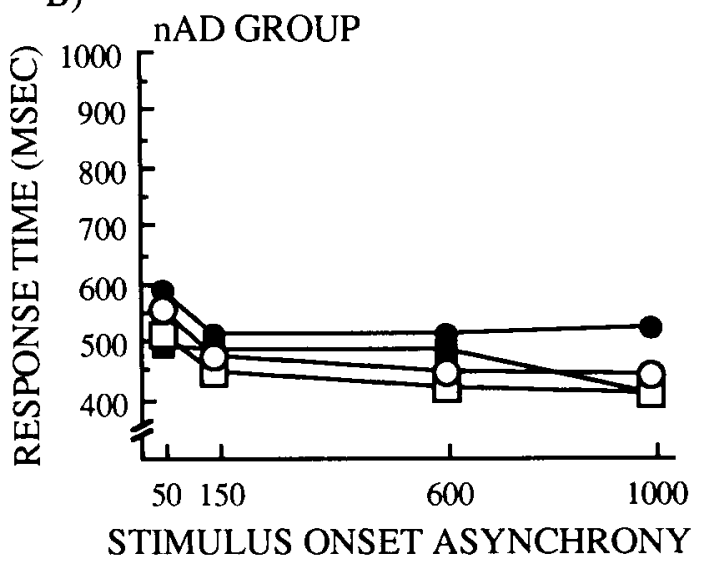

C)

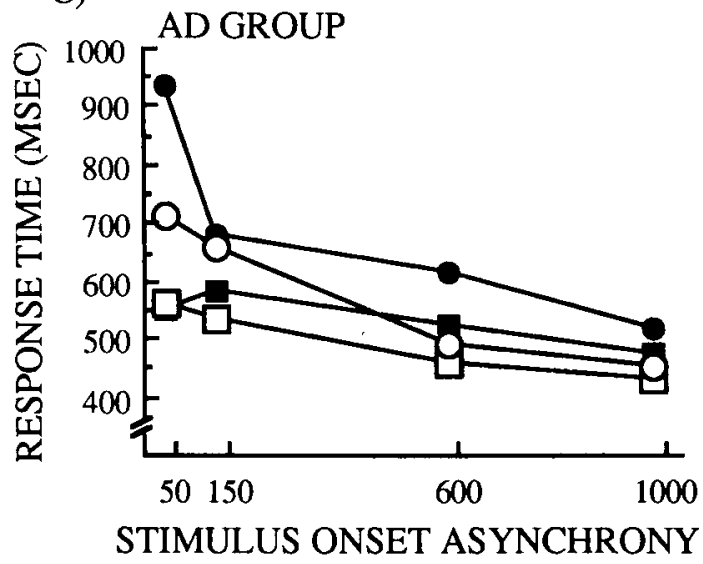

Figure 4. Mean response times observed in Experiment 1 (central cue). (A) Control group. (B) nAD group. (C) AD group. Square $=$ left-hemifield target. Circle $=$ right-hemifield target. Empty symbols $=$ valid-cue trials. Filled symbols $=$ invalid-cue trials.

Among the interactions that tested for specific categories of attention impairments, that of group $x$ hemifield was significant. Follow-up analyses of this interaction revealed no difference between the control and nAD groups on the effect of target hemifield, whereas such a difference did occur between the control and $\mathrm{AD}$ groups. No hemifield effect was observed in the normal controls but RTs were longer for right-hemifield targets in the AD group.

This result was qualified, however, by a significant group $\times$ hemifield $\times$ SOA interaction. Its decomposition revealed no difference between the normal controls and the $\mathrm{nAD}$ group on the conjoined effect of target hemifield and SOA. In contrast, the control and AD groups differed on the effect of target hemifield at short SOAs (50 and $150 \mathrm{msec}$ ). This discrepancy in the effect of hemifield between control and $\mathrm{AD}$ subjects was no longer significant at longer SOAs (600 and $1,000 \mathrm{msec}$ ).

The nature of the performance difference between the normal controls and AD subjects can be seen clearly in Figure 4. At short SOAs, and especially at $50 \mathrm{msec}$, the $\mathrm{AD}$ group showed delayed $\mathrm{RT}$ s for right-hemifield (contralesional) targets, an effect that was not apparent in the normal controls. At longer SOAs, the hemifield asymmetry in the $\mathrm{AD}$ group was markedly reduced and nonsignificant, and thus did not differ from the results observed in the controls.

Note that this variation of the hemifield effect as a function of SOA in the AD group includes the selection criterion upon which this group was formed. Obviously, this effect was expected for valid-cue trials, since the AD subjects were selected so that they would show it. It must be underlined however that a comparable pattern of results can be seen with targets preceded by invalid cues (Figure 4C). In fact, with an SOA of $50 \mathrm{msec}$ between the cue and the target, the RT increase for right-hemifield (contralesional) targets in the AD group is significantly larger with invalid than with valid cues $[F(1,17)=13.7$, $p<.005]$.

One important aspect of the results of Experiment 1 is the effect of cue validity, where RTs were shorter with a valid cue than with an invalid cue. This validity effect

Table 2

Analyses of Variance on the Data From the Central Cuing Experiment (Experiment 1)

\begin{tabular}{|c|c|c|c|}
\hline Interaction/Simple Effect & $d f$ & $F$ & $\underline{p}$ \\
\hline Cue validity & 1,17 & 55.2 & $<.001$ \\
\hline SOA & 3,15 & 30.0 & $<.001$ \\
\hline $\mathbf{G} \times \mathbf{H}$ & 2,17 & 13.5 & $<.001$ \\
\hline Controls vs. nAD on $\mathrm{H}$ & 1,17 & 3.5 & n.s. \\
\hline Controls vs. $\mathrm{AD}$ on $\mathrm{H}$ & 1,17 & 27.0 & $<.001$ \\
\hline $\mathrm{H}$ in controls & 1,17 & $<1$ & n.s. \\
\hline $\mathrm{H}$ in $\mathrm{AD}$ & 1,17 & 47.2 & $<.001$ \\
\hline $\mathbf{G} \times \mathbf{V}$ & 2,17 & 1.5 & n.s. \\
\hline $\mathbf{G} \times \mathbf{H} \times \mathbf{V}$ & 2,17 & 2.4 & n.s. \\
\hline $\mathbf{G} \times \mathbf{H} \times \mathbf{S}$ & 2,17 & 14.4 & $<.001$ \\
\hline Controls vs. $\mathrm{nAD}$ on $\mathrm{H} \times \mathrm{S}$ & 1,17 & $<1$ & n.s. \\
\hline Controls vs. $\mathrm{AD}$ on $\mathrm{H} \times \mathrm{S}$ & 1,17 & 25.6 & $<.001$ \\
\hline Controls vs. AD on $\mathrm{H}$ at short SOAs & 1,17 & 39.5 & $<.001$ \\
\hline H at short SOAs in controls & 1,17 & $<1$ & n.s. \\
\hline $\mathrm{H}$ at short SOAs in AD & 1,17 & 65.1 & $<.001$ \\
\hline Controls vs. AD on $\mathrm{H}$ at long SOAs & 1,17 & 3.0 & n.s. \\
\hline $\mathbf{G} \times \mathbf{V} \times \mathbf{S}$ & 2,17 & $<1$ & n.s. \\
\hline $\mathbf{G} \times \mathbf{H} \times \mathbf{V} \times \mathbf{S}$ & 2,17 & 1.0 & n.s. \\
\hline
\end{tabular}

Note-G, group; $H$, target hemifield; V, cue validity; $S$, stimulus onset asynchrony (SOA). 
demonstrates that subjects shifted their focus of visuospatial attention toward the location indicated by the central cue prior to the onset of the target.

Second, the results also show a reduction of RTs with increases in the temporal interval between the cue and the target. This effect has been observed on numerous occasions in the spatial cuing paradigm and is an indication that the onset of the cue also acts as a warning signal that produces an increase in the subject's preparation to respond to external stimulation (i.e., alerting).

The other statistical tests conducted on the data of Experiment 1 were aimed at identifying performance patterns in either of the brain-damaged groups that would correspond to a particular category of attention impairment. No significant difference between the control and nAD groups was revealed by these analyses. In contrast, the AD group exhibited results that diverged from those of the normal controls.

First, the AD group was shown to have longer RTs with right-hemifield (contralesional) than with left-hemifield (ipsilesional) targets. As indicated previously, this result may serve as preliminary evidence of a deficit in engaging the focus of attention. However, unambiguous support for an engagement deficit would have required that the RT increase for contralesional targets in the AD group be roughly constant across SOAs. This condition was not verified, since, as indicated above, the delayed RTs for contralesional targets in the AD group actually occurred only at short SOAs (50 and $150 \mathrm{msec}$ ) between the cue and the target.

From this, it appears that the anomaly shown by the AD group largely corresponds to the performance pattern expected in the case of an alerting impairment for contralesional stimulation. Thus, their RTs with contralesional targets were delayed at short temporal intervals (50 and $150 \mathrm{msec}$ ) following the cue, whereas this hemifield asymmetry was no longer present at longer SOAs (600 and $1,000 \mathrm{msec})$. In addition, this result occurred not only on valid-cue trials - that is, the data set on the basis of which the AD group was constituted-but also on invalid-cue trials.

However, there remains one aspect of the results of the AD group that cannot be directly accounted for by the hypothesis of an alerting impairment. It was mentioned above that, at a 50-msec SOA, the RTs to contralesional targets in the $\mathrm{AD}$ group were significantly more delayed, relative to ipsilesional targets, with invalid than with valid cues. Although this observation is not supported by a significant group $\times$ hemifield $\times$ validity $\times$ SOA interaction, it nevertheless seems that cue validity affected the deficit of the $\mathrm{AD}$ group for contralesional targets at short temporal intervals between the cue and target. More specifically, it appears that, at short SOAs, AD subjects experienced difficulty in reorienting their focus of attention toward the contralesional location if selective attention had just been miscued to an ipsilesional location. This kind of difficulty is comparable to that reported previously by Morrow and Ratcliff (1988) in right-brain-damaged pa- tients with visual hemineglect. They defined it as a refractory deficit in disengaging their attention from a contralesional location; that is, "if attention has been engaged to the ipsilateral side, a certain time interval ... must elapse before the disengage operation can be initiated" (Morrow \& Ratcliff, 1988, p. 268).

This result raises the possibility that $\mathrm{AD}$ subjects do not suffer from any impairment in attentional alerting and that their results in Experiment 1 can be entirely explained on the basis of a refractory disengagement disorder. This hypothesis readily accounts for the performance of the AD group with invalid central cues. In addition, it must also be noted that, with valid central cues, a disengagement operation may have been required before subjects could shift their attention toward the cued location. Indeed, it has previously been argued by Posner et al. (1984) that subjects must focus their attention on the central cue before directing it to the location it indicates. If this is correct, the delayed RTs shown by the AD group with valid central cues that preceded contralesional targets by a short SOA may be explained by their difficulty in disengaging their attention from the location occupied by the cue.

Alternatively, a discussion by Posner and colleagues (Posner, Inhoff, et al., 1987; see also Posner, 1990) of the relationships between different attentional subsystems suggests that the hypothesis of a primary alerting impairment in the AD group can be maintained. Indeed, on the basis of empirical observations, these authors have proposed that a deficit in alerting may retard the commands that are necessary to activate the system that is responsible for the disengagement of attention. Assuming that this hypothesis is correct, one may consider that the refractory disengagement difficulty seen in the AD group is actually a necessary consequence of their alerting impairment. Thus, it may be suggested that, in a poorly alerted state (i.e., at short SOAs), the damaged left hemisphere of the AD patients was unable to pull their focus of attention away from either an invalidly cued ipsilesional location or from the location of the central cue as rapidly as when it was in a higher state of alertness (i.e., at long SOAs).

In themselves, the results of Experiment 1 do not allow to distinguish between the two competing accounts for the attention deficit shown by the $\mathrm{AD}$ group with central cues. However, the results of Experiment 2 provide crucial evidence for this distinction.

Experiment 2: Motion cue. RTs observed in the control, $\mathrm{nAD}$, and $\mathrm{AD}$ groups with a motion cue are illustrated in Figure 5, and a summary of the data analyses is presented in Table 3.

As in the previous experiment, a significant main effect of SOA was found. This effect can be seen in Figure 5, where RTs decrease with increases in the time interval between the cue and target. However, contrary to the findings of Experiment 1, no RT difference was observed between valid and invalid motion cues.

The interaction of group $\times$ hemifield was significant and took the same form as that seen in the previous experiment. This effect was qualified, however, by a group 


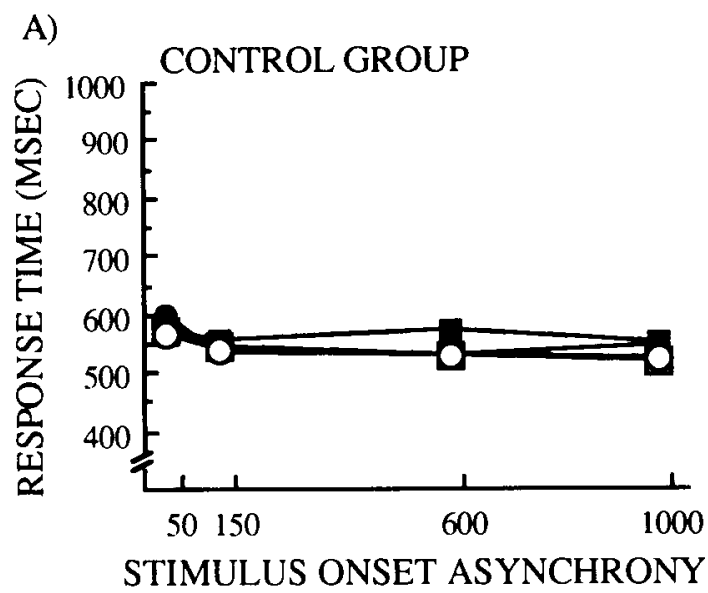

B)

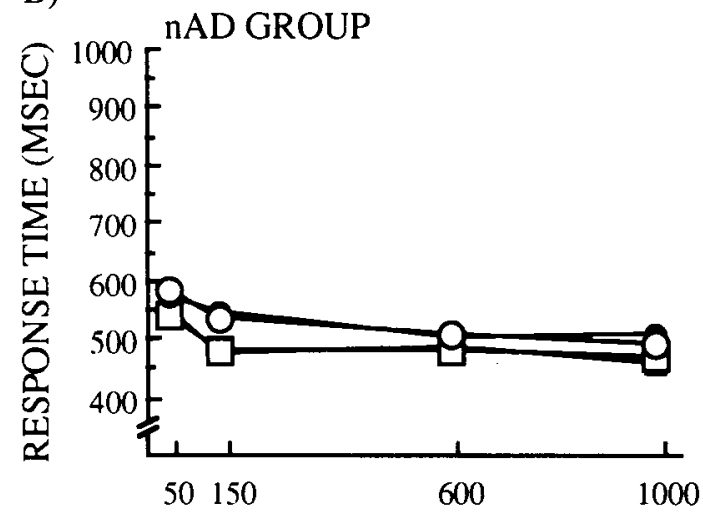

STIMULUS ONSET ASYNCHRONY

C)

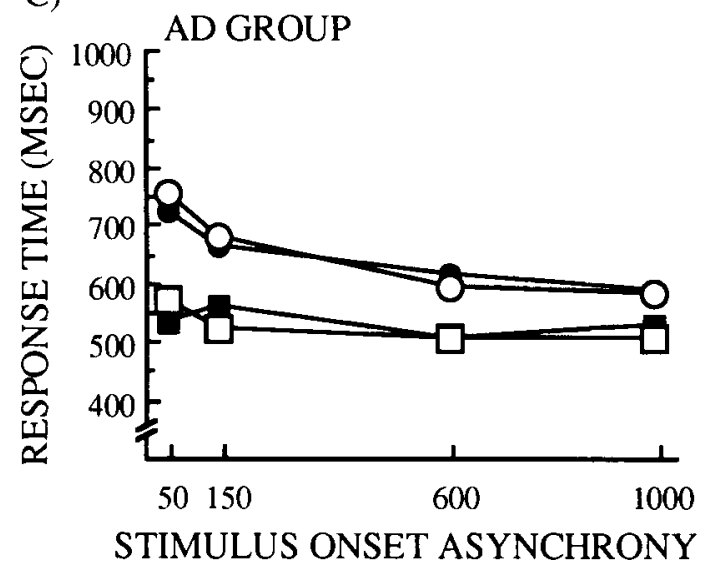

Figure 5. Mean response times observed in Experiment 2 (motion cue). (A) Control group. (B) nAD group. (C) AD group. Square = left-hemifield target. Circle $=$ right-hemifield target. Empty symbols $=$ valid-cue trials. Filled symbols $=$ invalid-cue trials.

$\times$ hemifield $\times$ SOA interaction. Simple effects performed on this interaction indicated that the combined effect of target hemifield and of SOA did not differ between the control and $\mathrm{nAD}$ groups. In contrast, such a difference did occur between the control and AD groups; that is, these two groups differed from one another on the variations of RTs across hemifield and SOA conditions. A follow-up on this result showed that normal controls and AD subjects differed on the effect of hemifield at both short (50- and 150-msec) and long (600- and 1,000-msec) SOAs, although the magnitude of this effect appeared weaker with long time intervals between the cue and target. At both short and long SOAs, the control group showed no RT difference between hemifields but the AD group exhibited longer RTs to contralesional targets.

The hemifield difference observed in the AD group, even though present across all SOAs, was largest at short SOAs (at $50 \mathrm{msec}$, the asymmetry is $189 \mathrm{msec}$ ) and monotonically decreased with longer SOAs (at $1,000 \mathrm{msec}$, the asymmetry is $69 \mathrm{msec}$ ). Although this result was expected on valid-cue trials because it was the basis for the selection of the AD group, it must be noted that the same variation in hemifield differences as a function of SOA was observed with invalid motion cues.

As was the case in the previous experiment, the results of Experiment 2 indicate that increases in the temporal interval between the cue and target led to a reduction in RTs. It therefore appears that the motion cue acted as a warning signal that alerted the subjects as to the imminence of the target, thus increasing their preparation to respond to it.

One major difference between the results of Experiments 1 and 2 is that, in the latter, the effect of cue validity was not significant. This indicates that, contrary to our expectations, the subjects did not use the information provided by the motion cues to direct their attention toward the most probable future target location. To the best of our knowledge, spatial cuing with motion stimuli such as those used here has not been reported before. The apparent incapacity of a direction of motion signal to serve as a

Table 3

Analyses of Variance on the Data From the Motion Cuing Experiment (Experiment 2)

\begin{tabular}{|c|c|c|c|}
\hline Interaction/Simple Effect & $d f$ & $F$ & $p$ \\
\hline Cue validity & 1,17 & 2.1 & n.s. \\
\hline SOA & 3,15 & 21.1 & $<.001$ \\
\hline $\mathbf{G} \times \mathbf{H}$ & 2,17 & 14.6 & $<.001$ \\
\hline Controls vs. $\mathrm{nAD}$ on $\mathrm{H}$ & 1,17 & 3.6 & n.s. \\
\hline Controls vs. AD on $\mathrm{H}$ & 1,17 & 29.2 & $<.001$ \\
\hline $\mathrm{H}$ in controls & 1,17 & $<1$ & n.s. \\
\hline $\mathrm{H}$ in $\mathrm{AD}$ & 1,17 & 39.6 & $<.001$ \\
\hline$G \times V$ & 2,17 & $<1$ & n.s. \\
\hline $\mathbf{G} \times \mathbf{H} \times \mathbf{V}$ & 2,17 & $<1$ & n.s. \\
\hline $\mathbf{G} \times \mathbf{H} \times \mathbf{S}$ & 2,17 & 6.2 & $<.01$ \\
\hline Controls vs. $\mathrm{nAD}$ on $\mathrm{H} \times \mathrm{S}$ & 1,17 & $<1$ & n.s. \\
\hline Controls vs. $A D$ on $H \times S$ & 1,17 & 12.1 & $<.005$ \\
\hline Controls vs. AD on $\mathrm{H}$ at short SOAs & 1,17 & 38.2 & $<.001$ \\
\hline $\mathrm{H}$ at short SOAs in controls & 1,17 & $<1$ & n.s. \\
\hline $\mathrm{H}$ at short SOAs in $\mathrm{AD}$ & 1,17 & 55.8 & $<.001$ \\
\hline Controls vs. AD on $\mathrm{H}$ at long SOAs & 1,17 & 7.1 & $<.01$ \\
\hline $\mathrm{H}$ at long SOAs in controls & 1,17 & $<1$ & n.s. \\
\hline $\mathrm{H}$ at long SOAs in $\mathrm{AD}$ & 1,17 & 16.8 & $<.005$ \\
\hline $\mathrm{G} \times \mathrm{V} \times \mathrm{S}$ & 2,17 & $<1$ & n.s. \\
\hline $\mathbf{G} \times \mathbf{H} \times \mathbf{V} \times \mathbf{S}$ & 2,17 & 2.0 & n.s. \\
\hline
\end{tabular}

Note-G, group; H, target hemifield; V, cue validity; $S$, stimulus onset asynchrony (SOA). 
usable location indicator may prove to be an interesting topic of investigation in future research on the interactions between separate visual subsystems that are specialized for the processing of particular stimulus dimensions. For the immediate purpose, however, the exact reason for this result is not clear, and it is beyond the scope of the present paper to speculate about it. The critical implication of the lack of a cue validity effect in Experiment 2 is that although motion cues affected alerting, as indicated by the effect of SOA on RTs, they had no impact on the spatial allocation of attention. In other words, it appears that motion cues were functionally equivalent to warning signals that provide no information about the future target location.

The last result of importance in Experiment 2 is the difference between normal controls and the AD group on the conjoined effect of hemifield and SOA between the cue and the target. As reported above, the control group showed no evidence for a difference in RT between hemifields. In contrast, the AD group exhibited markedly longer RTs for contralesional targets at short SOAs and an important but gradual reduction of this hemifield asymmetry as SOA increased. This effect of SOA on hemifield asymmetry was the same with valid and invalid motion cues. This pattern of performance is entirely congruent with the previously established diagnostic for an alerting impairment for contralesional stimulation. Moreover, and contrary to the results of Experiment 1, it does not appear that rival hypotheses about the nature of the attention deficit of $\mathrm{AD}$ subjects can account for these observations.

Even though the $\mathrm{AD}$ group showed longer $\mathrm{RT}$ s to contralesional targets across all SOAs, it appears unlikely that an impairment in attentional engagement can account for their results. Thus, a deficit in engaging attention to a contralesional target should not vary as a function of the temporal interval that separates a cue and the target. However, the results of Experiment 2 indicate that such a variation did occur (cf. the group $\times$ hemifield $\times$ SOA interaction).

In addition, it does not appear that the hypothesis of a disengagement disorder can provide an account of the performance of the AD group in Experiment 2. Indeed, motion cues did not affect the allocation of spatial attention-that is, no cue validity effect-and they were presented in the visual periphery rather than at fixation. This implies that there was no condition in which subjects had to disengage their attention from a particular location before directing it to the target. From this, it may be concluded that attentional disengagement was not a factor in Experiment 2. Therefore, if attentional disengagement had been the primary attention impairment in the AD group, they should have shown a normal performance in Experiment 2, which they did not.

From this discussion, it appears that the most likely account for the effect of SOA on the hemifield asymmetry shown by the AD group in Experiment 2 consists of delayed alerting for contralesional stimulation. Thus, at the shortest SOAs following the onset of the warning signal, the level of alertness of AD subjects for contralesional stimuli is lower than that for ipsilesional stimulation. The discrepancy in the level of alertness between hemifields then progressively decreases as an asymptotic alerting state is reached for ipsilesional targets at later SOAs.

The attention deficit suffered by the AD group may be assumed to remain constant across experiments. From the data of Experiment 1, two equally plausible conclusions were proposed. One was that the primary impairment of the AD subjects was one of attentional alerting, which mainly affected their RTs to contralesional stimulation and also had an impact on their capacity to disengage their attention from an invalidly cued ipsilesional location. The alternative was that $A D$ subjects suffered exclusively from a disengagement deficit. As argued above, the latter account does not seem to apply to the results of the AD group in Experiment 2. It may therefore be concluded that the primary impairment of the AD subjects is one of attentional alerting. This hypothesis can explain the main features of their anomalous performance in both Experiments 1 and 2 . The rival accounts examined here appear incapable of doing so.

The magnitude of the RT asymmetries exhibited by the $\mathrm{AD}$ group at the shortest SOAs is quite comparable across Experiments 1 and 2 . This is congruent with the view that the delayed alerting hypothesis may account for their deficit in these two experiments. Thus, with motion cues, RTs (averaged over valid- and invalid-cue trials) for contralesional targets were longer than those for ipsilesional targets by 189 and $130 \mathrm{msec}$ at SOAs of 50 and $150 \mathrm{msec}$, respectively. In comparison, the corresponding hemifield differences with valid central cues were 155 and $125 \mathrm{msec}$. It appears preferable not to include the invalid central cue trials in this comparison since an additional phenomenon, involving attentional disengagement, seems to have occurred in this condition, as proposed in the discussion of Experiment 1.

Experiment 3: Luminance cue. RTs observed with a luminance cue in the control, $\mathrm{nAD}$, and $\mathrm{AD}$ groups are presented in Figure 6. A summary of the analyses of variance is presented in Table 4.

The results of Experiment 3 revealed significant effects of cue validity and of temporal interval between cue and target. The validity effect indicated shorter $\mathrm{RTs}$ with valid than with invalid cues; the effect of SOA showed decreasing RTs with increasing time interval between the cue and target.

The group $\times$ validity interaction was significant, and pointed to a difference between the normal controls and the $\mathrm{AD}$ group on the magnitude of the effect of cue validity. Both groups showed a significant effect of cue validity. However, it can be seen in Figure 6 that the effect of cue validity was much larger in the $A D$ group $(110 \mathrm{msec}$ effect) than in the control group ( $32 \mathrm{msec}$ effect). The nAD group did not differ significantly from the normal controls on the cue validity effect.

A significant group $\times$ hemifield interaction was also observed. The effect of hemifield did not differ between 


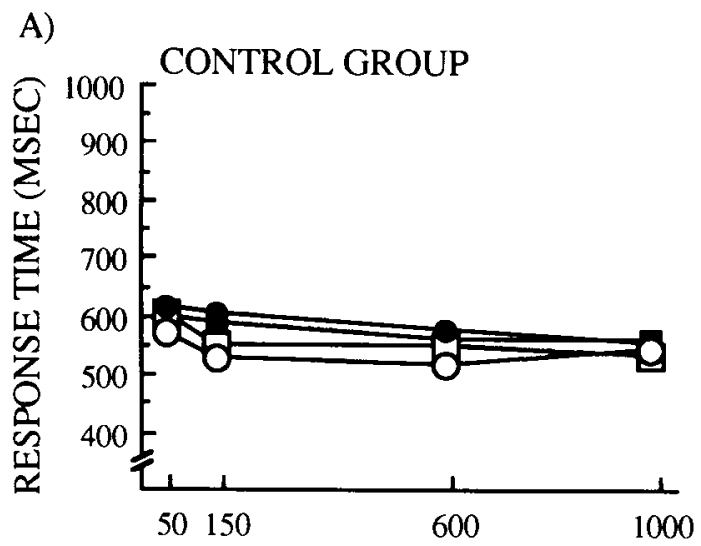

STIMULUS ONSET ASYNCHRONY

B)

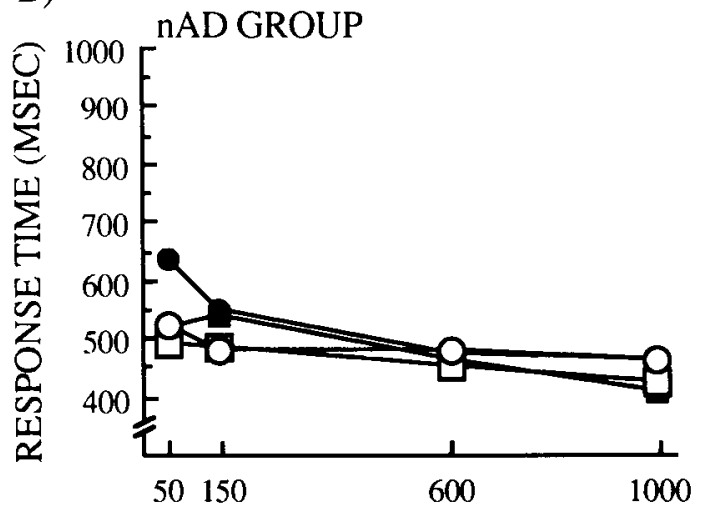

STIMULUS ONSET ASYNCHRONY

C)

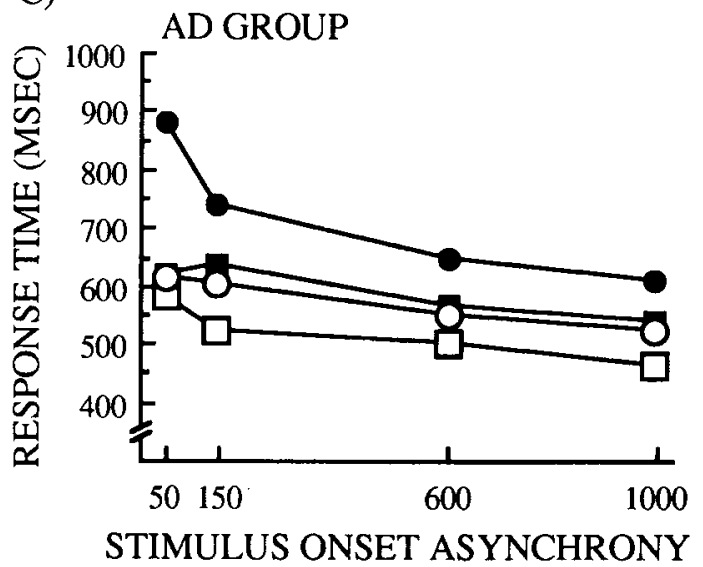

Figure 6. Mean response times observed in Experiment 3 (luminance cue). (A) Control group. (B) nAD group. (C) AD group. Square $=$ left-hemifield target. Circle $=$ right-hemifield target. Empty symbols $=$ valid-cue trials. Filled symbols $=$ invalid-cue trials.

the controls and the $\mathrm{nAD}$ group. In contrast, the $\mathrm{AD}$ group showed a large hemifield effect on RTs which was not present in the normal controls.

This last interaction was qualified by a significant group $x$ hemifield $\times$ SOA interaction. Normal controls and
nAD subjects did not differ on the hemifield $\times$ SOA effect, but such a difference did occur between the control and AD groups. At both short and long SOAs, no RT difference between hemifields was observed in the normal controls. In contrast, the AD group showed longer RTs with contralesional targets across all SOAs, although this difference was notably weaker at longer intervals (short SOAs, $119 \mathrm{msec}$; long SOAs, $65 \mathrm{msec}$ ).

The fact that the group $\times$ hemifield $\times$ validity $\times$ SOA interaction was not significant suggests that the variation of hemifield asymmetry as a function of SOA that was observed in the AD group was the same with valid and invalid cues. This, however, is in marked contrast with the results of the individual data analyses for Experiment 3, which showed that none of the brain-damaged subjects presented evidence for an attention deficit with valid luminance cues. This is also incongruent with what may be seen in Figure 6C. Thus, evidence of a decrease of hemifield asymmetry with increases in SOA in the AD group is only apparent for invalid-cue trials. This impression is substantiated by a significant hemifield $\times$ validity $\times$ SOA interaction in the AD group $[F(1,17)=9.00$; $p<.01]$. It appears, therefore, that the divergence exhibited by the AD group relative to the normal controls on the combined effect of target hemifield and SOA actually occurred exclusively with invalid cues in Experiment 3. As indicated above, this anomaly consisted in markedly longer RTs with contralesional targets at short time intervals between the invalid cue and the target and a decrease of this hemifield asymmetry at longer SOAs.

A first important point to note in the results of Experiment 3 is the significant effect of cue validity. This indi-

Table 4

Analyses of Variance on the Data From the Luminance Cuing Experiment (Experiment 3)

\begin{tabular}{|c|c|c|c|}
\hline Interaction/Simple Effect & $d f$ & $F$ & $p$ \\
\hline Cue validity & 1,17 & 71.0 & $<.00$ \\
\hline SOA & 3,15 & 16.4 & $<.00$ \\
\hline $\mathbf{G} \times \mathbf{H}$ & 2,17 & 8.5 & $<.00$ \\
\hline Controls vs. $\mathrm{nAD}$ on $\mathrm{H}$ & 1,17 & 2.9 & n.s. \\
\hline Controls vs. AD on $\mathrm{H}$ & 1,17 & 16.9 & $<.00$ \\
\hline $\mathbf{H}$ in controls & 1,17 & $<1$ & n.s. \\
\hline $\mathrm{H}$ in $\mathrm{AD}$ & 1,17 & 23.4 & $<.00$ \\
\hline $\mathbf{G} \times \mathbf{V}$ & 2,17 & 15.0 & $<.00$ \\
\hline Controls vs. $\mathrm{nAD}$ on $\mathrm{V}$ & 1,17 & $<1$ & n.s. \\
\hline Controls vs. AD on V & 1,17 & 26.7 & $<.00$ \\
\hline $\mathrm{V}$ in controls & 1,17 & 13.8 & $<.00$ \\
\hline $\mathrm{V}$ in $\mathrm{AD}$ & 1,17 & 80.2 & $<.00$ \\
\hline $\mathbf{G} \times \mathbf{H} \times \mathbf{V}$ & 2,17 & 1.8 & n.s. \\
\hline $\mathbf{G} \times \mathbf{H} \times \mathbf{S}$ & 2,17 & 4.5 & $<.05$ \\
\hline Controls vs. $n A D$ on $H \times S$ & 1,17 & $<1$ & n.s. \\
\hline Controls vs. $\mathrm{AD}$ on $\mathrm{H} \times \mathrm{S}$ & 1,17 & 8.7 & $<.01$ \\
\hline Controls vs. AD on $\mathrm{H}$ at short SOAs & 1,17 & 19.5 & $<.00$ \\
\hline $\mathrm{H}$ at short SOAs in controls & 1,17 & $<1$ & n.s. \\
\hline$H$ at short SOAs in $A D$ & 1,17 & 26.9 & $<.00$ \\
\hline Controls vs. $\mathrm{AD}$ on $\mathrm{H}$ at long SOAs & 1,17 & 4.8 & $<.05$ \\
\hline $\mathrm{H}$ at long SOAs in controls & 1,17 & $<1$ & n.s. \\
\hline $\mathrm{H}$ at long SOAs in $\mathrm{AD}$ & 1,17 & 13.0 & $<.00$ \\
\hline $\mathbf{G} \times \mathbf{V} \times \mathbf{S}$ & 2,17 & 1.9 & n.s. \\
\hline $\mathbf{G} \times \mathbf{H} \times \mathbf{V} \times \mathbf{S}$ & 2,17 & 1.8 & n.s. \\
\hline
\end{tabular}

Note-G, group; H, target hemifield; V, cue validity; S, stimulus onset asynchrony (SOA). 
cates that the luminance cue used here was effective in directing the subjects' attention to its location. As was the case in the previous experiments, a significant effect of SOA was also observed. It reflected decreasing RTs with increases in the temporal interval separating cue and target. This indicates that the luminance cue, more than just acting as a spatial signal, also acted as a warning that alerted the subjects to the imminent onset of the target.

The main aspect by which the $\mathrm{AD}$ group differed from normal controls was the large RT increase for contralesional targets with invalid cues and short SOAs, especially at $50 \mathrm{msec}$. This hemifield difference was markedly reduced at longer cue-to-target intervals. Taken in isolation, without consideration to the observations from Experiments 1 and 2, this result can be considered as congruent with the diagnostic pattern for a refractory disengagement deficit. According to this interpretation, AD subjects have difficulty in disengaging their attention from an invalidly cued ipsilesional location and then shifting it to a contralesional target. This would be true in cases where attention has been engaged at the invalid-cue location for a brief period of time-that is, short SOAs-but the deficit would be less apparent at the longer SOAs used here.

If one considers the observations gathered from the previous experiments, however, it seems that another interpretation must be provided for the results of the $\mathrm{AD}$ group with invalid luminance cues. Indeed, this set of results is also congruent with the diagnostic pattern for an alerting impairment for contralesional stimulation. As discussed previously, this hypothesis about the nature of the attention deficit of AD subjects appears as the more appropriate account for their results in Experiments 1 and 2. In particular, the observations from Experiment 2, where spatial cues were functionally equivalent to warning signals that provided no location information about the incoming target, ruled out an explanation of their deficit as being one of disengagement. Because the nature of the attention deficit of any brain-damaged subject is unlikely to vary as a function of experiment, a more parsimonious account of the performance exhibited by the AD group across the three experiments reported here must rest on the hypothesis that their primary attentional impairment is one of alerting.

In the attempt to provide a unitary account of the attention deficit of the AD subjects, we have previously compared the magnitudes of the hemifield asymmetries they showed with valid central cues and with both valid and invalid motion cues. These magnitudes were quite comparable, thus supporting the suggestion that a common process was at work. The same can be done here with the hemifield asymmetries seen in the AD group at short SOAs with invalid central and luminance cues. It must be recalled that, in Experiment 1, the hemifield asymmetry observed at a 50-msec SOA with invalid central cues was notably larger than that seen with valid central cues. At that point, it was suggested that this difference could possibly result from the effect of a lowered alerting state in the damaged hemisphere on the capacity of sub- jects to disengage their attention from an invalidly cued ipsilesional location (see also Posner, Inhoff, et al., 1987, and Posner, 1990). If this explanation is correct, a comparable phenomenon should have occurred in Experiment 3 with invalid luminance cues. This appears to be true, since a difference of $260 \mathrm{msec}$ occurred between the contralesional and ipsilesional hemifields, with invalid luminance cues preceding the target by an SOA of $50 \mathrm{msec}$. This asymmetry is larger than that observed with valid or invalid motion cues or with valid central cues.

In conclusion, a close examination of the results with valid and invalid central and motion cues as well as with invalid luminance cues provides support for the hypothesis of delayed alerting for contralesional stimulation in the AD group. This lowered alerting state appears to affect the capacity of subjects to disengage their attention from an invalidly cued ipsilesional target.

A different process seems to have occurred with valid luminance cues, however. Indeed, in this condition, the AD subjects exhibited longer RTs with contralesional targets, but this asymmetry remained fairly constant across all cue-to-target intervals. One reason why the results of the $\mathrm{AD}$ group with valid luminance cues may have been different from those found in other conditions is that only in this condition was the warning signal-the cue-presented unilaterally in the visual periphery and in the same hemifield as the target. Thus, central cues were shown at the point of ocular fixation, motion cues were presented bilaterally, and invalid luminance cues were displayed unilaterally but in the hemifield opposite the target. There is evidence that indicates that unilateral signals may have a special status with respect to the alerting, or cerebral activation, produced. Indeed, EEG as well as behavioral studies have shown that unilateral visual stimulation primarily produces activation of the hemisphere that is contralateral to the stimulated hemifield (Davidson et al., 1985; Reuter-Lorenz et al., 1990). In the case of valid contralesional luminance cues, it is possible that this lateralized activation produced by the unilateral signal had a very rapid effect on the low alertness level of the damaged hemisphere in the AD group, and therefore led to a reduced hemifield asymmetry in RTs even at the earliest SOAs between the cue and target.

\section{FEATURES OF THE BRAIN DAMAGE ASSOCIATED WITH AN ATTENTION DEFICIT}

An attempt was made to establish a relationship between the alerting deficit exhibited by subjects of the AD group and the features of their brain lesions. This anatomicalclinical correlation was performed here with the use of CT scans, which were available for all the brain-damaged subjects in our sample. Two main parameters were considered: the locus of the lesion and its volume.

To investigate the effect of the localization of brain damage associated with the attention deficit of the $A D$ group, we attempted to specify a lesional site common 


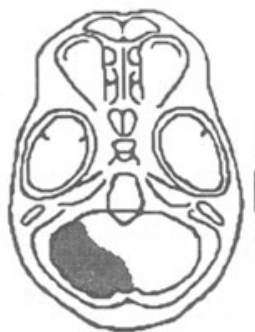

SLICE 1
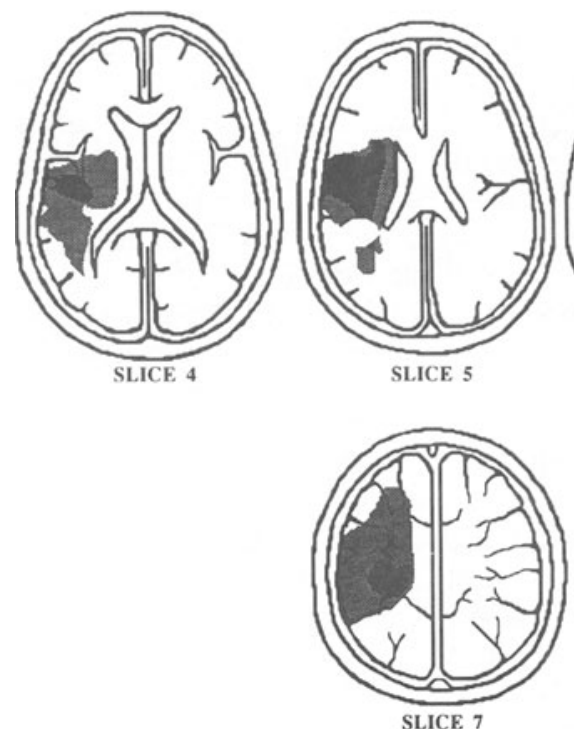

Figure 7. Analysis of the lesional sites responsible for the attention deficit observed in AD subjects. The gray areas represent lesional sites observed in only $1 \mathrm{AD}$ subject, and black areas represent lesional sites seen in $\mathbf{2}$ AD subjects. See text for details on the construction of this figure.

to all these subjects while excluding lesional sites observed in the nAD group. Figure 7 presents the results of such an analysis. The first step in making Figure 7 was to superimpose reconstructions of the lesional sites observed in the subjects from the AD group. Second, to exclude lesional sites that were not associated with the deficit present in the $\mathrm{AD}$ group, the loci that were common to the $\mathrm{AD}$ and $\mathrm{nAD}$ groups were removed from this representation. The effectiveness of this subtraction process was limited by the small size of our sample. Nevertheless, it appeared that the procedure increased the possibility of specifying a particular locus associated with the attention deficit shown by group $\mathrm{AD}$.

As may be seen in Figure 7, it was not possible to specify a single lesional site as responsible for the attention deficit observed in the AD group. There were indeed important differences in the lesional sites observed in the AD subjects. Thus, Subject P9 showed a cerebellar lesion, which may be seen in Slices 1 and 2 of Figure 7. In contrast, Subject P6 showed subcortical damage in the area of the internal capsule. This lesion may be seen in the median portion of Slice 3 . Finally, the other 3 AD sub- jects (P7, P8, and P10) all showed damage at the temporoparieto-occipital junction (Slices 3-7). Still, this apparent concentration cannot easily be considered as associated with the attention deficit observed in AD subjects, since Subject P5, who did not show evidence of an alerting disorder, also had a lesion in that area. This can be seen in Figure 7 by noting the white spot that separates in two distinct parts the locus that is illustrated in Slice 5. It therefore appears that the analysis of the brain lesions showed by $\mathrm{AD}$ subjects did not establish a clear correlation between their attention deficit and a specific brain area.

A second feature of the brain damage that was examined in the brain-damaged subjects of our sample was the volume of the lesion. This parameter was assessed, for each brain-damaged subject, on the basis of our reconstruction of his/her lesion. The measurement unit used was arbitrary, but the scale remained constant across subjects. Table 5 presents the results of this analysis. On the average, this volumetric index was nearly four times larger in the $\mathrm{AD}$ group than it was in the $\mathrm{nAD}$ group. This result suggests that a mass effect rather than lesion localization may be the most plausible anatomical account for the alerting deficit observed in the AD subjects. ${ }^{4}$ Considering the size of our sample, however, this conclusion must remain tentative. Thus, it remains possible, if not likely, that the lesion size effect actually reflects the likelihood that a distributed neural network responsible for behavioral alerting is involved.

\section{DISCUSSION}

The main observation reported in this paper concerns the effect of SOA between a warning signal-the spatial cues-and target onset on the change in RT asymmetries between hemifields in a subgroup of left-brain-damaged patients (P6-P10; AD subjects). Thus, in all the cuing conditions used here, with the exception of valid luminance cues, these subjects showed a marked hemifield asymmetry-longer RTs to a contralesional (right hemifield) target - that diminished or even disappeared with increasing SOAs. This pattern of hemifield effect as a function of SOA was not observed with neurologically intact controls. The performance pattern exhibited by the AD subjects is congruent with the diagnostic for a deficit affecting the time course of alerting for contralesional stimulation. These results thus suggest that, at the shortest

Table 5

Volume of the Lesion Observed in Each Brain-Damaged Subject

\begin{tabular}{crrrr}
\hline \multicolumn{3}{c}{ Group } & \multicolumn{2}{c}{} \\
\cline { 1 - 2 } \cline { 5 - 5 } Subject & NAD & & AD \\
\cline { 5 - 5 } P1 & 273.7 & & P6 & Volume \\
P2 & 86.9 & & P7 & 52.8 \\
P3 & 72.3 & & P8 & 50.0 \\
P4 & 83.6 & & P9 & 90.0 \\
P5 & 39.0 & & P10 & 1079.0 \\
Avg. & 111.1 & & & 413.7 \\
\hline
\end{tabular}


delays following the onset of the cues/warning signals ( 50 and $150 \mathrm{msec}$ ), the damaged hemisphere of AD subjects was in a lower state of alertness than was the intact hemisphere. However, at longer SOAs (600 and 1,000 msec), after the state of alertness of the intact hemisphere of the AD subjects had reached asymptote, that of the damaged hemisphere continued to grow, which thus led to a reduction in the RT asymmetries between the hemifields.

The lateralized alerting deficit described here was not evident in all brain-damaged cases, however, for Subjects P1-P5 (nAD subjects) showed a performance that did not differ significantly from that of the normal controls. Analysis of the properties of the brain damage suffered by the nAD and AD subjects suggested that volume of the damage may be the main factor responsible for the alerting deficit in the latter group. Thus, the average volume of the lesions seen in the AD patients was almost four times higher than that observed in the $\mathrm{nAD}$ patients. It should be recalled, however, that lesion size may in fact reflect the probability of damage to a distributed neural substrate that is critical for alerting.

The findings presented here are at variance with those reported by Posner, Inhoff, et al. (1987). Indeed, those authors found an alerting impairment in a no-cue condition in their right-brain-damaged subjects but not in those with left hemisphere lesions. What are the reasons for this discrepancy between our results and those of Posner, Inhoff, et al.? Several methodological differences exist between these two sets of experiments and it appears reasonable to suggest that one or a combination of them must be responsible for the discrepant observations.

The most obvious difference is that whereas the deficit observed here in the AD patients affected the time course of alerting, that observed by Posner, Inhoff, et al. (1987) was one in which subjects were unable to maintain a proper alert state in the time interval between trials. Therefore, it may be suggested that only measures that are sensitive to the time course of alerting of the damaged hemisphere are able to reveal a deficit in patients with left-brain damage. It should be noted, though, that even if a no-cue (or 0-msec SOA) condition was not used in the present experiments, it is conceivable that the asymmetry in the level of alertness shown by the $\mathrm{AD}$ subjects at SOAs of $50 \mathrm{msec}$ is the reflection of a similar asymmetry before the onset of the warning signal.

Another possibility is related to the size of the lesion suffered by the left-brain-damaged patients studied by Posner, Inhoff, et al. (1987). As indicated earlier, the impairment reported here as to the time course of alerting does not seem to have affected all our patients, and the volume of damaged brain tissue appears to be a factor in the occurrence of the deficit. It is conceivable that the lesions suffered by the left-brain-damaged subjects of Posner, Inhoff, et al. did not possess the critical feature responsible for an alerting impairment.

Finally, Posner, Inhoff, et al. (1987) did not examine the details of the performance of individual patients. It therefore remains possible that some of the patients they studied showed a deficit whereas others did not, and that indications of an alerting impairment in some subjects were lost when the data were averaged across several cases.

\section{Peripheral Warning Signals and Alerting}

One cuing condition in which the $A D$ group seemed free of a hemifield asymmetry in the time course of alerting was that with valid luminance cues. These signals have a property unique among the variety of cuing stimuli used in the present experiments. Specifically, they were shown unilaterally in the visual periphery and in the same hemifield as the incoming target. It thus seems that the onset of this type of signal in the contralesional hemifield of the AD subjects produced a very rapid increase in the level of preparation of the damaged hemisphere for the processing of the target, thus reducing the hemifield asymmetry in RTs in comparison with other types of warning signals. As mentioned earlier, this appears congruent with previous observations indicating that the alerting property of unilateral signals primarily affects the contralateral hemisphere.

Another puzzling result in Experiments 2 and 3 is related to the persistence of the hemifield asymmetries in RTs in the AD group, which remained present even after $1,000 \mathrm{msec}$ following the onset of the cues. Two alternative accounts of this effect can be suggested.

One is based on a report by Rafal and Posner (1987) that revealed a deficit in engaging attention on a contralesional target in patients with unilateral thalamic damage. However, it was indicated before that the conclusion of an attentional engagement deficit requires that the hemifield asymmetry exhibited by the subjects of interest remain constant across SOAs. Neither the results of Experiment 2 nor those of Experiment 3 meet this condition. More importantly, perhaps, the persistence of longer contralesional RTs at an SOA of $1,000 \mathrm{msec}$ in the AD group was not observed in Experiment 1. Thus, in this condition, AD subjects' RTs were quite similar for ipsilesional and for contralesional targets. If $\mathrm{AD}$ subjects truly suffered from a disorder affecting the engagement of attention on a contralesional target, one would expect this deficit to appear consistently.

Another account for the longer RTs to contralesional targets at an SOA of 1,000 msec in the AD group in Experiments 2 and 3 is that warning signals presented in the visual periphery are not powerful enough to entirely compensate for these patients' alerting deficit. Certain points tend to support this hypothesis. One is that the persistent hemifield asymmetry was not observed in Experiment 1, in which the warning signal (i.e., the central cue) was presented at fixation, whereas it was present in both experiments in which warning signals were peripheral. Also, one should remember that subjects were instructed to maintain fixation at the center of the display for the trials to begin and that careful eye monitoring was performed. Given this constraint in maintaining a proper eye fixation, it is possible that subjects also focused their atten- 
tion at the location of the fixation point. If so, the difference between a central cue and cues presented in the periphery in their alerting potential may have resulted from the fact that subjects focused their attention at the location of the central cue but not at locations where peripheral cues were displayed. This account is quite speculative, though, and further experiments should be conducted to determine whether warning signals presented at an attended location provide a more potent alerting effect than warnings presented at other locations.

\section{Alerting and the Spatial Allocation of Attention}

As pointed out in the discussion of the results of Experiment 2 (motion cues), the hypothesis of a refractory disengagement disorder cannot account for the overall pattern of performance of the AD subjects across experiments. However, it appears that symptoms analogous to such a refractory disengagement deficit may have appeared as a consequence of the alerting impairment in the AD group. Thus, in both Experiments 1 and 3, where a cue validity effect was observed, if the cue was invalid and it preceded the target by an SOA of $50 \mathrm{msec}$, the hemifield difference in RTs shown by the AD subjects was much larger than that seen in any other cuing condition in any of the three experiments. This suggests that, when in a state of low alertness, the damaged hemisphere of the AD patients is unable to rapidly pull the focus of attention away from the invalidly cued ipsilesional location in order to orient it toward the contralesional target location.

Such an interaction between the capacity to disengage attention and the level of alertness is congruent with a hypothesis made by Posner, Inhoff, et al. (1987; see also Posner, 1990) about the interrelationships between different attentional subsystems. They suggested that deficits in alerting may retard the commands that are necessary to activate the system that is responsible for the disengagement of attention from one location in order to shift it toward another location. Our proposal is also consistent with a larger view of alerting (Posner, 1978), which suggests that alerting does not influence the buildup of perceptual information as such but rather affects the rate at which a high-level attention system may respond to external stimulation.

\section{Conclusions}

The observations reported here suggest a deficit leading to a delay in the effect of an alerting stimulus for the processing of contralesional stimulation in a subset of leftbrain-damaged patients (AD subjects). The occurrence of this impairment appears to be related to the size of the brain damage suffered by the patients. The results also indicate that, when the damaged hemisphere of these $A D$ subjects is in a poorly alerted state (i.e., at an SOA of $50 \mathrm{msec}$ ), the disengagement of attention away from an ipsilesional invalidly cued location is delayed in comparison with when the level of alertness is higher. Finally, it appears that unilateral peripheral warning signals have a very rapid effect on the level of alertness for the processing of contralesional stimulation in AD subjects, although the potential of peripheral warning stimuli-or of those outside the focus of attention-to reduce the hemispheric asymmetry in the level of alertness appears to be weaker than those presented at fixation-or those inside the focus of attention.

\section{REFERENCES}

ARGUIN, M., \& BUB, D. (1993). Modulation of the directional attention deficit in visual neglect by hemispatial factors. Brain \& Cognition, 22, 148-160.

Baynes, K., Holtzman, J. D., \& Volpe, B. T. (1986). Components of visual attention: Alteration in response pattern to visual stimuli following parietal lobe infarction. Brain, 109, 99-114.

BERTELSON, P. (1967). The time course of preparation. Quarterly Journal of Experimental Psychology, 19, 272-279.

Davidson, R. J., SchafFer, C. E., \& SARON, C. (1985). Effects of lateralized stimulus presentation on the self-report of emotion and EEG asymmetry in depressed and non-depressed subjects. Psychophysiology, 22, 353-364.

De Renzi, E., \& FagLioni, P. (1965). The comparative efficiency of intelligence and vigilance tests detecting hemispheric change. Cortex, 1, 410-433.

Donchin, E., Coles, M. G. H., \& Gratton, G. (1984). Cognitive psychophysiology and preparatory processes: A case study. In S. Kornblum \& J. Requin (Eds.), Preparatory states and processes (pp. 155178). Hillsdale, NJ: Erlbaum.

EgLiN, M. (1987). The effects of different attentional loads on feature integration in the cerebral hemispheres. Perception \& Psychophysics, 42, 81-86.

Egly, R., \& Hoм^, D. (1984). Sensitization of the visual field. Journal of Experimental Psychology: Human Perception \& Performance, 10, 778-793.

Eriksen, C. W., \& Hoffman, J. E. (1973). The extent of processing of noise elements during selective encoding from visual displays. Perception \& Psychophysics, 14, 155-160.

ErIKSEN, C. W., HoffMan, J. E. (1974). Selective attention: Noise suppression or signal enhancement? Bulletin of the Psychonomic Society, 4, 587-589.

Friedman, A., Polson, M. C. (1981). Hemispheres as independent resource systems: Limited-capacity processing and cerebral specialization. Journal of Experimental Psychology: Human Perception \& Performance, 7, 1031-1058.

GaILlARD, A. W., \& NÄÄTÄNEN, R. (1973). Slow potential changes and choice reaction time as a function of interstimulus interval. Acta Psychologica, 37, 173-186.

Gauthier, L., Dehaut, F., \& Jonnette, Y. (1989). The bells test: A quantitative and qualitative test for visual neglect. International Journal of Clinical Neuropsychology, 11, 49-54.

Heilman, K. M. (1985). Neglect and related disorders. In K. M. Heilman \& E. Valenstein (Eds.), Clinical neuropsychology (2nd ed., pp. 243-293). New York: Oxford University Press.

Heilman, K. M., \& Van den Abell, T. (1979). Right hemispheric dominance for mediating cerebral activation. Neuropsychologia, 17, 315-321.

Heilman, K. M., \& VAN DEn Abell, T. (1980). Right hemisphere dominance for attention: The mechanism underlying hemispheric asymmetry of inattention. Neurology, 30, 327-330.

Hellige, J. B., Cox, P. J., \& Litvac, L. (1979). Information processing in the cerebral hemispheres: Selective hemispheric activation and capacity limitations. Journal of Experimental Psychology: General, 108, 251-279.

HiLlyard, S. A. (1969). Relationships between the contingent negative variation (CNV) and reaction time. Physiology \& Behavior, 4, 351-357. 
Hughes, H. C., Zimba, L. D. (1985). Spatial maps of directed visual attention. Journal of Experimental Psychology: Human Perception \& Performance, 11, 409-430.

JonIDES, J. (1980). Towards a model of the mind's eye's movement. Canadian Journal of Psychology, 34, 103-112.

JONIDES, J. (1981). Voluntary versus automatic control over the mind's eye's movement. In J. Long \& A. Baddeley (Eds.), Attention and performance $I X$ (pp. 187-203). Hillsdale, NJ: Erlbaum.

KinsBOURNE, M. (1970). A model for the mechanism of unilateral neglect. Transactions of the American Neurological Association, 95, 143-146.

KINSBOURNE, M. (1975). The mechanism of hemispheric control of the lateral gradient of attention. In P. M. A. Rabbit \& S. Dornic (Eds.), Attention and performance V (pp. 81-97). New York: Academic Press.

KinSBOURNE, M. (1977). Hemi-neglect and hemispheric rivalry. In E. A. Weinstein \& R. P. Friedland (Eds.), Hemi-inattention \& hemispheric specialization (pp. 41-52). New York: Raven Press.

KINSBOURNE, M. (1978). Asymmetrical function of the brain. Cambridge: Cambridge University Press.

KinsBourne, M. (1987). Mechanisms of unilateral neglect. In M. Jeannerod (Ed.), Neurophysiological and neuropsychological aspects of spatial neglect (pp. 69-86). New York: North-Holland.

Kinsbourne, M., \&icks, R. E. (1978). Functional cerebral space: A model for overflow transfer and interference effects in human performance. In J. Requin (Ed.), Attention and performance VII (pp. 8197). Hillsdale, NJ: Erlbaum.

KLEIN, R. (1980). Does oculomotor readiness mediate cognitive control of visual attention? In R. S. Nickerson (Ed.), Attention and performance VIII (pp. 259-276). Hillsdale, NJ: Erlbaum.

Lגdavas, E., Del Pesce, M., \& Provinciali, L. (1989). Unilateral attention deficits and hemispheric asymmetries in the control of visual attention. Neuropsychologia, 27, 353-366.

LANSING, R. W., Schwartz, E., \& Lindsley, D. B. (1959). Reaction time and EEG activation under alerted and non-alerted conditions. Journal of Experimental Psychology, 58, 1-7.

Morrow, L. A., \& RATCLIFF, G. (1988). The disengagement of covert attention and the neglect syndrome. Psychobiology, 16, 261-269.

MÜlleR, H. J., \& RABBITT, P. M. A. (1989). Reflexive and voluntary orienting of visual attention: Time course of activation and resistance to interruption. Journal of Experimental Psychology: Human Perception \& Performance, 15, 315-330.

O'Brien, R. G., \& KAISER, M. K. (1985). MANOVA method for analyzing repeated measures designs: An extensive primer. Psychological Bulletin, 97, 316-333.

OLDFELD, O. D. (1971). The assessment and analysis of handedness: The Edinburgh Inventory. Neuropsychologia, 9, 97-113.

Petersen, S. E., Robinson, D. L., \& Morris, J. D. (1987). Contributions of the pulvinar to visual spatial attention. Neuropsychologia, 25, 97-105.

POSNER, M. I. (1978). Chronometric explorations of mind. Hillsdale, NJ: Erlbaum.

Posner, M. I. (1980). Orienting of attention. Quarterly Journal of Experimental Psychology, 32, 3-25.

POSNER, M. I. (1990). Hierarchical distributed networks in the neuropsychology of selective attention. In A. Caramazza (Ed.), Cognitive neuropsychology and neurolinguistics: Advances in models of cognitive function and impairment (pp. 187-210). Hillsdale, NJ: Erlbaum.

Posner, M. I., \& Bores, S. J. (1971). Components of visual attention. Psychological Review, 78, 391-408.

Posner, M. I., Cohen, Y., \& RAfal, R. D. (1982). Neural systems of spatial orienting. Philosophical Transactions of the Royal Society of London: Series B, 298, 187-198.

PoSNer, M. I., INHOFF, A. W., FrIEDRICH, F. J., \& COHEN, A. (1987). Isolating attentional systems: A cognitive-anatomical analysis. Psychobiology, 15, 107-121.

Posner, M. I., Klein, R., Summers, J., Buggie, S. (1973). On the selection of signals. Memory \& Cognition, 1, 2-12.

Posner, M. I., Nissen, M. J., \& OGDEN, W. C. (1978). Attended and unattended processing modes: The role of set for spatial location. In H. L. Pick \& I. J. Saltzman (Ed.), Modes of perceiving and processing information (pp. 137-157). Hillsdale, NJ: Erlbaum.
Posner, M. I., Rafal, R. D., Choate, L. S., Vaughan, J. (1985). Inhibition of return: Neural basis and function. Cognitive Neuropsychology, 2, 211-228.

Posner, M. I., Walker, J. A., Friedrich, F. J., \& Rafal, R. D. (1984). Effects of parietal injury on covert orienting. Joumal of Neuroscience, 4, 1863-1874.

Posner, M. I., Walker, J. A., Friedrich, F. A., Rafal, R. D (1987). How do the parietal lobes direct covert attention? Neuropsychologia, 25, 135-145.

Pribram, K. H., McGuinness, D. (1975). Arousal activation and effort in the control of attention. Psychological Review, 182, 116-149.

RAFAl, R. D., \& Posner, M. I. (1987). Deficits in human visual spatial attention following thalamic lesions. Proceedings of the National Academy of Sciences, 84, 7349-7353.

Rafal, R. D., Posner, M. I., Friedman, J. H., Inhoff, A. W., BerNSTEIN, E. (1988). Orienting of visual attention in progressive supranuclear palsy. Brain, 111, 267-280.

Reuter-Lorenz, P. A., Kinsbourne, M., Moscovitch, M. (1990). Hemispheric control of spatial attention. Brain \& Cognition, 12, 240-266.

Rizzolatti, G., Riggio, L., Dascola, I., \& Umilta, C. (1987). Reorienting attention across the horizontal and vertical meridians: Evidence in favor of a premotor theory of attention. Neuropsychologia, 25, 31-40.

SANDERS, A. F. (1972). Some remarks on mental load. In N. Moray (Ed.), Mental workload: Its theory and measurement (pp. 41-78). New York: Plenum.

Shulman, G. L., Remington, R. W., \& Mclean, J. P. (1979). Moving attention through visual space. Joumal of Experimental Psychology: Human Perception \& Performance, 5, 522-526.

Tassinari, G., Agliotti, S., Chelazzi, L., Marzi, C. A., \& BerLUCCHI, G. (1987). Distribution in the visual field of the costs of voluntarily allocated attention and of the inhibitory after-effects of covert orienting. Neuropsychologia, 25, 55-71.

Thомаs, E. (1974). The selectivity of preparation. Psychological Review, 81, 442-464.

Tucker, P. M., \& Williamson, P. A. (1984). Asymmetric neural control in human self-regulation. Psychological Review, 91, 185-215.

Walter, W. G., Cooper, R., Aldridge, V. J., McCallum, W. C., \& WINTER, A. L. (1964). Contingent negative variation: An electrical sign of sensorimotor association and expectancy in the human brain. Nature, 203, 380-384.

\section{NOTES}

1. Six similarly selected right-brain-lesioned subjects were also examined. However, only 1 of them could be assessed satisfactorily, since the others tended to fall asleep after a few minutes into the experiment. This is probably related to the vigilance and alertness problems which are frequently associated with right-brain damage. The only rightbrain-damaged subject who could be tested performed similarly to the left-brain-damaged individuals who, here, do not show any indication of an alerting deficit. Since he was the only subject with a right-brain lesion in our sample, his results will not be reported.

2 . These numerical labels were used solely for convenience of presentation and are in no way related to the chronological order in which patients were tested.

3. We checked to ensure that the abnormal performance pattern revealed by the group data analyses in the AD subjects (described below) was not simply an artifact of the procedure with which this group was selected. To do this, the comparisons listed in Table 1 were conducted between the five normal controls who showed the lowest measure of asymmetry (low $M$; see Equation 1) for a particular experiment and those who showed the highest (high $M$ ). None of the features that characterized the abnormal performance of the AD group were observed in those comparisons between low- and high- $M$ normal controls.

4. The AD and nAD groups did not differ from each other in time elapsed between the onset of brain damage and testing $[t(8)=0.19$, n.s. $]$.

(Manuscript received October 19, 1992; revision accepted for publication July 21,1993 .) 